\title{
The Galactic WC and WO stars
}

\section{The impact of revised distances from Gaia DR2 and their role as massive black hole progenitors}

\author{
A. A. C. Sander ${ }^{1,2}$, W.-R. Hamann ${ }^{1}$, H. Todt $^{1}$, R. Hainich ${ }^{1}$, T. Shenar ${ }^{1,3}$, V. Ramachandran ${ }^{1}$, and L. M. Oskinova ${ }^{1}$ \\ ${ }^{1}$ Institut für Physik und Astronomie, Universität Potsdam, Karl-Liebknecht-Str. 24/25, 14476 Potsdam, Germany \\ e-mail: ansander@astro.physik.uni-potsdam.de \\ 2 Armagh Observatory and Planetarium, College Hill, Armagh BT61 9DG, UK \\ 3 Institute of Astrophysics, KU Leuven, Celestijnenlaan 200 D, 3001 Leuven, Belgium
}

Received 25 June 2018 / Accepted 26 November 2018

\begin{abstract}
Wolf-Rayet stars of the carbon sequence (WC stars) are an important cornerstone in the late evolution of massive stars before their core collapse. As core-helium burning, hydrogen-free objects with huge mass-loss, they are likely the last observable stage before collapse and thus promising progenitor candidates for type $\mathrm{Ib} / \mathrm{c}$ supernovae. Their strong mass-loss furthermore provides challenges and constraints to the theory of radiatively driven winds. Thus, the determination of the WC star parameters is of major importance for several astrophysical fields. With Gaia DR2, for the first time parallaxes for a large sample of Galactic WC stars are available, removing major uncertainties inherent to earlier studies. In this work, we re-examine a previously studied sample of WC stars to derive key properties of the Galactic WC population. All quantities depending on the distance are updated, while the underlying spectral analyzes remain untouched. Contrasting earlier assumptions, our study yields that WC stars of the same subtype can significantly vary in absolute magnitude. With Gaia DR2, the picture of the Galactic WC population becomes more complex: We obtain luminosities ranging from $\log L / L_{\odot}=4.9-6.0$ with one outlier (WR 119) having $\log L / L_{\odot}=4.7$. This indicates that the WC stars are likely formed from a broader initial mass range than previously assumed. We obtain mass-loss rates ranging between $\log \dot{M}=-5.1$ and -4.1 , with $\dot{M} \propto L^{0.68}$ and a linear scaling of the modified wind momentum with luminosity. We discuss the implications for stellar evolution, including unsolved issues regarding the need of envelope inflation to address the WR radius problem, and the open questions in regard to the connection of WR stars with Gamma-ray bursts. WC and WO stars are progenitors of massive black holes, collapsing either silently or in a supernova that most-likely has to be preceded by a WO stage.
\end{abstract}

Key words. stars: evolution - stars: mass-loss - stars: Wolf-Rayet - stars: massive - stars: distances - Galaxy: stellar content

\section{Introduction}

Classical Wolf-Rayet (WR) stars are descendants of evolved, massive stars that have lost their outermost layers. Officially defined in a purely spectroscopic way by the presence of certain emission lines in their optical spectrum (e.g. Hiltner \& Schild 1966; Smith 1968a; Crowther et al. 1998), the term "classical" was added more recently to distinguish them from other objects with similar emission-line phenomena, e.g. central stars of planetary nebulae or the so-called O-stars on steroids, in other words very massive early-type stars with strong winds that are still in the stage of core-hydrogen burning. Based on the presence of prominent emission lines of nitrogen or carbon, WR stars are sorted into the nitrogen (WN), carbon (WC), and oxygen (WO) sequence with subclasses defined by the ratios of characteristic emission lines. While some WN stars have considerable amounts of hydrogen, all known WC and WO stars do not reveal any measurable amount of hydrogen in their spectra.

The WO subtype is defined by the (strong) presence of O vi $\lambda 3811 \AA$ (compared to O v $\lambda 5590 \AA$ ) that is usually seen either as an extension of the WC sequence to hotter temperatures or a distinct spectroscopic sequence of more evolved stars. Current evolutionary calculations (e.g. Groh et al. 2014) and empirical studies (e.g. Sander et al. 2012; Tramper et al. 2015) support a picture that combines both approaches: Late WO subtypes have chemical compositions quite similar to early WC subtypes while early WO subtypes have higher oxygen abundances and indeed reflect the last stage before core collapse that is observable for a perceptible duration of time.

Wolf-Rayet stars have an enormous influence on their environment. Due to their high temperatures, they produce large numbers of ionizing photons. Their huge mass-loss enriches the surrounding interstellar medium with metals and injects a significant amount of mechanical energy into it. Few WR stars can equal or even outweigh the influence of a whole population of nearby OB stars (e.g. Ramachandran et al. 2018). Thus, quantifying their impact is of particular importance for a variety of astrophysical applications.

As hydrogen-free and thus (at least) core-helium burning objects, the WC and WO stars represent a clearly defined stage. In Sander et al. (2012), all (putatively) single Galactic WC and WO stars with at that time available spectra were analyzed with the help of the Potsdam Wolf-Rayet (PoWR) model atmospheres. To obtain their luminosities, their distances need to be known. While this is not much of an issue for WR populations in other galaxies (see, e.g. Hainich et al. 2014, 2015; Sander et al. 2014; Shenar et al. 2016), most of the distances to the WR stars in our Milky Way were not well constrained before Gaia. They are too far away to have a (reliable) HIPPARcos parallax, and especially the unobscured sources are often relatively isolated. Thus 
only a small sub-sample in Sander et al. (2012) had distances that were due to confirmed or suspected cluster or association membership. However, even for confirmed cluster members, the distances were not always robust, as also the distances to various clusters had been a matter of debate, as for example reflected in the discussion about the Be 87 cluster hosting the WO2 star WR 142 (cf. appendix A in Sander et al. 2012).

Roughly two decades after HipParcos, the Gaia mission (Gaia Collaboration 2016), now with its second data release (Gaia Collaboration 2018), provides for the first time parallaxes (Luri et al. 2018) for most of the unobscured Galactic WR stars, covering the full sample of Galactic WC stars from Sander et al. (2012). Based on these parallaxes, new distances can be derived and we can revise the luminosities and further distance-dependent parameters such as mass-loss rates. In this work, we only focus on the impact of the revised distances and do not perform any updates of our atmosphere models assigned to each of the sample stars. While the PoWR code has undergone considerable extensions during the last years (e.g. Shenar et al. 2014; Sander et al. 2015, 2017), these are either not essential for the empirical analysis of WR stars at Galactic metallicity or have only moderate effects on the resulting stellar parameters. Thus, instead of confusing the effects of some model details with the impact of the revised distances, we decided to focus on the latter.

In contrast, the PoWR models compiled in the online library ${ }^{1}$ contain the most recent grids of WC models for Galactic and LMC metallicity, accounting for the updates and extensions of the PoWR code in the past years. Besides many minor improvements and additional information on the website, the most prominent update compared to the WC grid released with Sander et al. (2012) is an improved treatment of the superlevels for the iron group elements and the addition of infrared line spectra to the online database. While the deduced stellar parameters for the Galactic WC sample with the newer generation of models are within the uncertainty of what we deduced with the older generation, the improved treatment of the iron group elements can have a considerable effect on parts of the emergent spectrum for certain parameter regimes, especially in the UV. Thus, for a detailed study of a particular WC star, we recommend to use the newest version of the WC grid.

Based on recent literature, we re-examined the binary status of each object in our sample. WR 14 was reported to exhibit significant variability in the radio domain, which was suggested to originate from binarity (Drissen et al. 1992; De Becker \& Raucq 2013). However, the spectral analysis compared to other stars of the same subtype does not indicate a significant contribution from the companion.

It is generally thought that many if not all of the dustproducing WC9 stars are members in (long-period) binaries (e.g. Usov 1991; Tuthill et al. 1999). For WR 65, the new distance leads to an X-ray luminosity of roughly $L_{\mathrm{X}} \approx 10^{33} \mathrm{erg} \mathrm{s}^{-1}$, a value typical for colliding wind binaries (CWBs), a suggestion already reported by Oskinova \& Hamann (2008). Desforges et al. (2017) investigated a large sample of WC9/WC9d stars for line-flux variability, but could not find evidence for periodicity in their data, with the possible exception of WR 103. However, they cannot exclude the possibility that the stars are members of long-period binaries. The WN/WC transition-type star WR 145 is a WR+O colliding wind binary system which unfortunately was not properly marked in Sander et al. (2012). For completeness, we keep it in our sample, but set a mark in Table 1 and also comment on it in Sect. 3.5.

http://www . astro.physik. uni-potsdam.de/PowR
Apart from WR 145, a few objects are suspicious and we discuss these in Sect. 3.5 and mark them in Table 1, meaning that their derived parameters have to be taken with care. For the remaining objects in our sample, we assume that they are either single or that their companion contributes only negligibly to the visual spectrum, thus not significantly affecting the derived parameters.

The structure of this paper is as follows: in Sect. 2, we briefly discuss the issue of converting parallaxes into distances and how the other updated quantities are calculated. The following Sect. 3 contains the results, sorted into subsections discussing the various aspects such as luminosities, mass-loss rates, or the absolute magnitudes of the sample stars. In Sect. 4, we put the revised results into an evolutionary context before eventually drawing the conclusions in Sect. 5. In a subsequent paper, we will perform a similar study for the Galactic WN stars.

\section{Impact of revised distances}

In principle the distance $d$ to a particular object is the inverse of its parallax $\varpi$. Unfortunately, this only holds for the "true" parallax, meaning if the measurement of $\varpi$ would have no or negligible errors. An inspection of $\sigma_{\varpi}$, the standard deviation of $\varpi$, also denoted as "standard error" in the Gaia data archive, reveals that while there are 12 sources for which the relative error $\sigma_{\varpi} / \varpi$ is less than 0.1 , most parallaxes have a larger uncertainty. Moreover, $\varpi$ itself is not a direct measurement, but the result of interpreting the observed change of direction to a source with the help of a timedependent model describing both the motion of the actual source through space as well as the motion of the observational instrument, that is in this case Gaia (see, e.g., Lindegren et al. 2016), around the sun. Thus the determined values of $\varpi$ are already the result from a fit procedure which, in the case of noisy observations, can lead to unphysical negative parallaxes. Even for positive values, the simple inversion of $d=\varpi^{-1}$ provides a poor distance estimate for $\sigma_{\varpi} / \varpi>0.1$, as discussed in detail in Bailer-Jones (2015) and, more recently, Luri et al. (2018).

For updating the distances of our Galactic WC and WO star sample, we thus decided to use the distances by Bailer-Jones et al. (2018). These are calculated from the Gaia DR2 parallaxes with the help of a Bayesian approach. They assume a prior with an exponentially decreasing space density in distance with a length-scale parameter $\ell$ that depends on Galactic latitude and longitude. Assuming a Gaussian likelihood for the parallax $\varpi$ as motivated by Lindegren et al. (2018), the distances given by Bailer-Jones et al. (2018) are then obtained by the mode of the posterior, which is derived by solving a thirdorder equation depending on $\ell$. The uncertainties for the distance are defined by the lower and upper boundaries of the highest density interval around the mode of the posterior with probability $p=0.6827$. For a Gaussian posterior, these would correspond to $\pm 1 \sigma$. To quantify the impact of these distance uncertainties, we will list corresponding error margins in Table 1 on all quantities directly depending on the distance.

In this work, we purely focus on the impact of distances and thus do not update any of the atmosphere models applied in Sander et al. (2012). This means in particular that the obtained temperature $T_{*}$ and the so-called "transformed radius"

$R_{\mathrm{t}}=R_{*}\left[\frac{v_{\infty}}{2500 \mathrm{~km} \mathrm{~s}^{-1}} / \frac{\dot{M} \sqrt{D}}{10^{-4} M_{\odot} \mathrm{yr}^{-1}}\right]^{\frac{2}{3}}$

(Schmutz et al. 1989; Hamann \& Koesterke 1998) remain the same. In Eq. (1), $R_{*}$ denotes the stellar radius which is also the inner boundary of the unterlying atmosphere models defined at 
Table 1. Parameters of the Galactic single WC stars.

\begin{tabular}{|c|c|c|c|c|c|c|c|c|c|c|c|c|c|}
\hline WR & Subtype & $\begin{array}{r}T_{*}^{a} \\
(\mathrm{kK}) \\
\end{array}$ & $\begin{array}{c}\log R_{\mathrm{t}}{ }^{a} \\
\left(R_{\odot}\right)\end{array}$ & $\begin{array}{l}v_{\infty}{ }^{a} \\
\left(\mathrm{~km} \mathrm{~s}^{-1}\right)\end{array}$ & $X_{\mathrm{He}}{ }^{a}$ & $\begin{array}{l}E_{b-v}^{a} \\
\text { (mag) }\end{array}$ & $\begin{array}{l}\text { D.M. }{ }^{b} \\
\text { (mag) }\end{array}$ & $\begin{array}{r}M_{v} \\
(\mathrm{mag}) \\
\end{array}$ & $\begin{array}{r}R_{*} \\
\left(R_{\odot}\right) \\
\end{array}$ & $\begin{array}{r}\log \dot{M} \\
\left(M_{\odot} \mathrm{yr}^{-1}\right) \\
\end{array}$ & $\begin{array}{l}\log L \\
\left(L_{\odot}\right)\end{array}$ & $\begin{array}{r}M^{c} \\
\left(M_{\odot}\right) \\
\end{array}$ & \\
\hline 102 & WO2 & 200 & 0.40 & 5000 & 0.30 & 1.08 & $12.14_{-0.16}^{+0.17}$ & $\rightarrow-1.46$ & 0.52 & $-5.23_{-0.05}^{+0.05}$ & $5.58_{-0.06}^{+0.07}$ & $16.1_{-1.4}^{+1.7}$ & 3.8 \\
\hline 142 & WO2 & 200 & 0.30 & 5000 & 0.30 & 1.43 & $11.09_{-0.10}^{+0.10}$ & $\rightarrow-3.13$ & 0.80 & $-4.80_{-0.03}^{+0.03}$ & $5.96_{-0.04}^{+0.04}$ & $28.6_{-1.8}^{+2.1}$ & 4.3 \\
\hline 38 & WC4 & 126 & 0.10 & 3200 & 0.55 & 1.11 & $14.22_{-0.31}^{+0.35}$ & $\rightarrow-3.36$ & 0.85 & $-4.66_{-0.09}^{+0.10}$ & $5.21_{-0.12}^{+0.14}$ & $10.4_{-1.4}^{+1.9}$ & 21.6 \\
\hline 52 & WC4 & 112 & 0.20 & 3225 & 0.35 & 0.56 & $11.22_{-0.14}^{+0.15}$ & $\rightarrow-3.21$ & 0.92 & $-4.75_{-0.04}^{+0.04}$ & $5.07_{-0.06}^{+0.06}$ & $8.5_{-0.5}^{+0.6}$ & 24.1 \\
\hline 144 & WC4 & 112 & 0.20 & 3500 & 0.35 & 1.60 & $11.24_{-0.19}^{+0.21}$ & $\rightarrow-3.28$ & 1.06 & $-4.62_{-0.06}^{+0.06}$ & $5.20_{-0.08}^{+0.08}$ & $9.9_{-0.9}^{+1.1}$ & 26.4 \\
\hline 4 & C5 & 79 & 0.50 & 2528 & 0.55 & 0.60 & $12.85_{-0.31}^{+0.35}$ & $\rightarrow-5.14$ & 3.85 & $-4.37_{-0.09}^{+0.11}$ & $5.71_{-0.12}^{+0.14}$ & $20.0_{-3.2}^{+4.6}$ & 10.3 \\
\hline 17 & WC5 & 79 & 0.50 & 2231 & 0.55 & 0.31 & $14.59_{-0.57}^{+0.65}$ & $\rightarrow-5.60$ & 4.01 & $-4.40_{-0.17}^{+0.20}$ & $5.74_{-0.23}^{+0.26}$ & $21.0_{-5.7}^{+10.5}$ & 7.8 \\
\hline 33 & WC5 & 79 & 0.50 & 3342 & 0.55 & 0.60 & $14.72_{-0.44}^{+0.49}$ & $\rightarrow-4.77$ & 3.25 & $-4.36_{-0.13}^{+0.15}$ & $5.56_{-0.18}^{+0.20}$ & $16.3_{-3.4}^{+5.2}$ & 19.5 \\
\hline 111 & WC5 & 89 & 0.40 & 2398 & 0.55 & 0.34 & $11.10_{-0.27}^{+0.31}$ & $\rightarrow-4.26$ & 2.10 & $-4.64_{-0.08}^{+0.09}$ & $5.39_{-0.11}^{+0.12}$ & $13.0_{-1.7}^{+2.3}$ & 11.0 \\
\hline 114 & WC5 & 79 & 0.50 & 3200 & 0.55 & 1.35 & $11.61_{-0.17}^{+0.18}$ & $\rightarrow-4.19$ & 2.68 & $-4.51_{-0.05}^{+0.05}$ & $5.39_{-0.07}^{+0.07}$ & $13.1_{-1.0}^{+1.3}$ & 19.7 \\
\hline 150 & WC5 & 89 & 0.40 & 3000 & 0.55 & 0.80 & $14.97_{-0.38}^{+0.41}$ & $\rightarrow-5.26$ & 3.59 & $-4.19_{-0.11}^{+0.12}$ & $5.86_{-0.15}^{+0.16}$ & $24.8_{-5.0}^{+7.4}$ & 13.3 \\
\hline 5 & WC6 & 79 & 0.50 & 2120 & 0.55 & 0.85 & $12.36_{-0.21}^{+0.23}$ & $\rightarrow-4.61$ & 3.12 & $-4.59_{-0.06}^{+0.07}$ & $5.53_{-0.08}^{+0.09}$ & $15.5_{-1.6}^{+2.1}$ & 7.9 \\
\hline 13 & WC6 & 79 & 0.50 & 2000 & 0.55 & 1.21 & $13.46_{-0.32}^{+0.36}$ & $\rightarrow-4.64$ & 3.28 & $-4.58_{-0.10}^{+0.11}$ & $5.57_{-0.13}^{+0.14}$ & $16.5_{-2.6}^{+3.7}$ & 6.9 \\
\hline 15 & WC6 & 79 & 0.50 & 2675 & 0.55 & 1.23 & $12.37_{-0.17}^{+0.19}$ & $\rightarrow-5.69$ & 5.31 & $-4.14_{-0.05}^{+0.06}$ & $5.99_{-0.07}^{+0.08}$ & $30.6_{-3.2}^{+4.1}$ & 9.7 \\
\hline 23 & C6 & 79 & 0.50 & 2342 & 0.55 & 0.55 & 12.0 & $\rightarrow-4.91$ & 3.43 & $-4.49_{-0.06}^{+0.07}$ & 5.6 & $17.4_{-1.8}^{+2.3}$ & 9.2 \\
\hline 27 & WC6 & 79 & 0.50 & 2100 & 0.55 & 1.40 & $12.13_{-0.18}^{+0.19}$ & $\rightarrow-3.92$ & 2.35 & $-4.78_{-0.05}^{+0.06}$ & $5.28_{-0.07}^{+0.08}$ & $11.3_{-0.9}^{+1.1}$ & 9.0 \\
\hline 45 & WC6 & 79 & 0.50 & 2200 & 0.55 & 1.44 & $13.20_{-0.15}^{+0.16}$ & $\rightarrow-4.30$ & 2.87 & $-4.63_{-0.04}^{+0.05}$ & $5.45_{-0.06}^{+0.06}$ & $14.1_{-1.0}^{+1.2}$ & 9.0 \\
\hline 132 & WC6 & 71 & 0.60 & 2400 & 0.55 & 1.15 & $13.16_{-0.24}^{+0.27}$ & $\rightarrow-4.38$ & 3.15 & $-4.67_{-0.07}^{+0.08}$ & $5.35_{-0.10}^{+0.11}$ & $12.4_{-1.4}^{+1.8}$ & 11.3 \\
\hline 154 & WC6 & 79 & 0.50 & 2300 & 0.55 & 0.78 & $13.74_{-0.36}^{+0.41}$ & $\rightarrow-5.59$ & 4.88 & $-4.26_{-0.11}^{+0.12}$ & $5.91_{-0.14}^{+0.16}$ & $27.2_{-5.3}^{+8.3}$ & 7.6 \\
\hline 14 & $\mathrm{C} 7$ & 71 & 0.60 & 2194 & 0.55 & 0.65 & $11.74_{-0.15}^{+0.16}$ & $\rightarrow-5.37$ & 5.16 & $-4.39_{-0.05}^{+0.05}$ & $5.78_{-0.06}^{+0.07}$ & $22.1_{-1.9}^{+2.3}$ & 7.3 \\
\hline 56 & WC7 & 71 & 0.60 & 2009 & 0.55 & 0.70 & $15.20_{-0.37}^{+0.41}$ & $\rightarrow-4.13$ & 3.09 & $-4.76_{-0.11}^{+0.12}$ & $5.33_{-0.15}^{+0.16}$ & $12.1_{-2.0}^{+2.8}$ & 7.9 \\
\hline 64 & $\mathrm{C} 7$ & 71 & 0.60 & 1700 & 0.55 & 1.20 & $15.02_{-0.42}^{+0.47}$ & $\rightarrow-4.35$ & 2.87 & $-4.88_{-0.13}^{+0.14}$ & $5.27_{-0.17}^{+0.19}$ & $11.2_{-2.1}^{+3.0}$ & 5.9 \\
\hline 68 & $\mathrm{C} 7$ & 7 & 0.60 & 2100 & 0.55 & 1.40 & $13.55_{-0.33}^{+0.38}$ & $\rightarrow-5.16$ & 4.96 & $-4.44_{-0.10}^{+0.11}$ & $5.74_{-0.13}^{+0.15}$ & $21.0_{-3.6}^{+5.4}$ & 6.8 \\
\hline 90 & $\mathrm{Cr}$ & 71 & 0.60 & 2053 & 5 & 0.40 & $10.31_{-0.14}^{+0.15}$ & $\rightarrow-4.99$ & 4.00 & $-4.59_{-0.04}^{+0.04}$ & $5.55_{-0.06}^{+0.06}$ & $16.2_{-1.2}^{+1.4}$ & 7.3 \\
\hline 53 & $\mathrm{C} 8 \mathrm{~d}$ & 50 & 0.90 & 1800 & 0.55 & 0.75 & $13.22_{-0.29}^{+0.33}$ & $\rightarrow-5.41$ & 7.77 & $-4.66_{-0.09}^{+0.10}$ & $5.52_{-0.12}^{+0.13}$ & $15.5_{-2.2}^{+3.1}$ & 5.8 \\
\hline 57 & WC8 & 63 & 0.70 & 1787 & 0.55 & 0.38 & $13.85_{-0.46}^{+0.54}$ & $\rightarrow-5.61$ & 6.36 & $-4.50_{-0.14}^{+0.16}$ & $5.75_{-0.18}^{+0.22}$ & $21.2_{-4.8}^{+8.4}$ & 4.9 \\
\hline 60 & WC8 & 63 & 0.70 & 2300 & 0.55 & 1.45 & $12.80_{-0.27}^{+0.31}$ & $\rightarrow-5.49$ & 6.77 & $-4.35_{-0.08}^{+0.09}$ & $5.80_{-0.11}^{+0.12}$ & $23.0_{-3.4}^{+4.8}$ & 8.0 \\
\hline 135 & WC8 & 6 & 0.60 & 1343 & 0.75 & 0.40 & $11.50_{-0.14}^{+0.15}$ & $\rightarrow-4.78$ & 4.24 & $-4.73_{-0.04}^{+0.04}$ & $5.40_{-0.06}^{+0.06}$ & $13.6_{-0.9}^{+1.1}$ & 4.9 \\
\hline $59^{d}$ & $9 \mathrm{~d}$ & 40 & 1.00 & 1300 & 0.55 & 2.00 & $12.87_{-0.29}^{+0.33}$ & $\rightarrow-7.31$ & 15.89 & $-4.48_{-0.09}^{+0.10}$ & $5.76_{-0.12}^{+0.13}$ & $21.4_{-3.3}^{+4.7}$ & 3.7 \\
\hline $65^{d}$ & WC9d & 40 & 1.00 & 1300 & 0.55 & 2.00 & $12.65_{-0.37}^{+0.43}$ & $\rightarrow-7.09$ & 18.50 & $-4.38_{-0.11}^{+0.13}$ & $5.89_{-0.15}^{+0.17}$ & $26.2_{-5.2}^{+8.5}$ & 3.4 \\
\hline 69 & WC9d & 40 & 1.00 & 1089 & 0.55 & 0.55 & $12.73_{-0.26}^{+0.29}$ & $\rightarrow-5.51$ & 9.77 & $-4.87_{-0.08}^{+0.09}$ & $5.33_{-0.10}^{+0.12}$ & $12.1_{-1.4}^{+1.9}$ & 3.3 \\
\hline 80 & WC9d & 45 & 0.90 & 1600 & 0.55 & 1.80 & $12.91_{-0.53}^{+0.65}$ & $\rightarrow-5.88$ & 6.89 & $-4.79_{-0.16}^{+0.20}$ & $5.24_{-0.21}^{+0.26}$ & $10.8_{-2.4}^{+4.2}$ & 7.5 \\
\hline 81 & WC9 & 45 & 0.80 & 1600 & 0.55 & 1.50 & $11.67_{-0.27}^{+0.31}$ & $\rightarrow-5.44$ & 7.08 & $-4.62_{-0.08}^{+0.09}$ & $5.26_{-0.11}^{+0.12}$ & $11.1_{-1.4}^{+1.8}$ & 10.4 \\
\hline 88 & WC9/WN8 & 40 & 1.00 & 1500 & 0.55 & 1.40 & $12.74_{-0.35}^{+0.41}$ & $\rightarrow-5.78$ & 11.73 & $-4.62_{-0.11}^{+0.12}$ & $5.49_{-0.14}^{+0.16}$ & $14.9_{-2.5}^{+3.7}$ & 5.7 \\
\hline 92 & WC9 & 45 & 0.80 & 1121 & 0.55 & 0.52 & $12.93_{-0.43}^{+0.52}$ & $\rightarrow-4.50$ & 4.97 & $-5.00_{-0.13}^{+0.16}$ & $4.95_{-0.17}^{+0.21}$ & $7.7_{-1.3}^{+2.1}$ & 6.1 \\
\hline 95 & WC9d & 45 & 0.90 & 1900 & 0.55 & 1.74 & $11.69_{-0.32}^{+0.37}$ & $\rightarrow-5.25$ & 6.86 & $-4.71_{-0.09}^{+0.11}$ & $5.23_{-0.13}^{+0.15}$ & $10.7_{-1.5}^{+2.1}$ & 10.8 \\
\hline 103 & WC9d & 45 & 0.80 & 1190 & 0.55 & 0.52 & $12.79_{-0.49}^{+0.61}$ & $\rightarrow-6.06$ & 9.31 & $-4.56_{-0.15}^{+0.18}$ & $5.50_{-0.19}^{+0.24}$ & $15.0_{-3.3}^{+6.0}$ & 5.1 \\
\hline 106 & WC9d & 45 & 0.80 & 1100 & 0.55 & 1.20 & $12.62_{-0.33}^{+0.38}$ & $\rightarrow-5.36$ & 6.81 & $-4.80_{-0.10}^{+0.11}$ & $5.23_{-0.13}^{+0.15}$ & $10.6_{-1.5}^{+2.2}$ & 5.1 \\
\hline 117 & WC9d & 56 & 0.60 & 2000 & 0.55 & 1.56 & $12.97_{-0.57}^{+0.70}$ & $\rightarrow-5.19$ & 5.12 & $-4.44_{-0.17}^{+0.21}$ & $5.36_{-0.23}^{+0.28}$ & $12.5_{-3.0}^{+5.5}$ & 15.5 \\
\hline 119 & WC9d & 45 & 0.80 & 1300 & 0.55 & 0.90 & $12.72_{-0.51}^{+0.62}$ & $\rightarrow-3.91$ & 3.70 & $-5.13_{-0.15}^{+0.19}$ & $4.70_{-0.20}^{+0.25}$ & $5.8_{-1.1}^{+1.8}$ & 9.6 \\
\hline 121 & WC9d & 45 & 0.80 & 1100 & 0.55 & 1.40 & $11.75_{-0.21}^{+0.24}$ & $\rightarrow-5.08$ & 6.35 & $-4.85_{-0.06}^{+0.07}$ & $5.16_{-0.09}^{+0.09}$ & $9.9_{-0.9}^{+1.2}$ & 5.3 \\
\hline 26 & WN7/WCE & 79 & 0.60 & 2700 & 0.78 & 1.25 & $14.26_{-0.24}^{+0.27}$ & $\rightarrow-4.74$ & 3.94 & $-4.29_{-0.07}^{+0.08}$ & $5.73_{-0.10}^{+0.11}$ & $21.1_{-2.6}^{+3.5}$ & 12.7 \\
\hline 58 & WN4/WCE & 79 & 0.50 & 1600 & 0.98 & 0.55 & $14.00_{-0.40}^{+0.46}$ & $\rightarrow-3.34$ & 1.61 & $-4.95_{-0.12}^{+0.14}$ & $4.95_{-0.16}^{+0.19}$ & $8.4_{-1.3}^{+1.9}$ & 9.9 \\
\hline 126 & WC5/WN & 63 & 1.20 & 2000 & 0.75 & 0.95 & $14.83_{-0.43}^{+0.49}$ & $\rightarrow-5.43$ & 9.23 & $-4.96_{-0.13}^{+0.15}$ & $6.07_{-0.17}^{+0.20}$ & $35.9_{-8.6}^{+15.2}$ & 0.9 \\
\hline $145^{e}$ & WN7/WCE & 50 & 0.90 & 1440 & 0.98 & 1.86 & $10.82_{-0.13}^{+0.14}$ & $\rightarrow-5.90$ & 8.59 & $-4.49_{-0.04}^{+0.04}$ & $5.61_{-0.05}^{+0.06}$ & $18.3_{-1.2}^{+1.4}$ & 5.6 \\
\hline
\end{tabular}

Notes. ${ }^{(a)}$ Unchanged from Sander et al. (2012). ${ }^{(b)}$ All distance moduli based on Gaia DR2 parallaxes converted into distances by Bailer-Jones et al. (2018). ${ }^{(c)}$ Masses are calculated from luminosity after Langer (1989) using their $M-L$ relation for WC stars. ${ }^{(d)}$ Binary candidate with possibly significant contribution from the secondary. See comments in Sect. 3.5. ${ }^{(e)}$ Binary system analyzed as a single source. See comments in Sect. 3.5. 


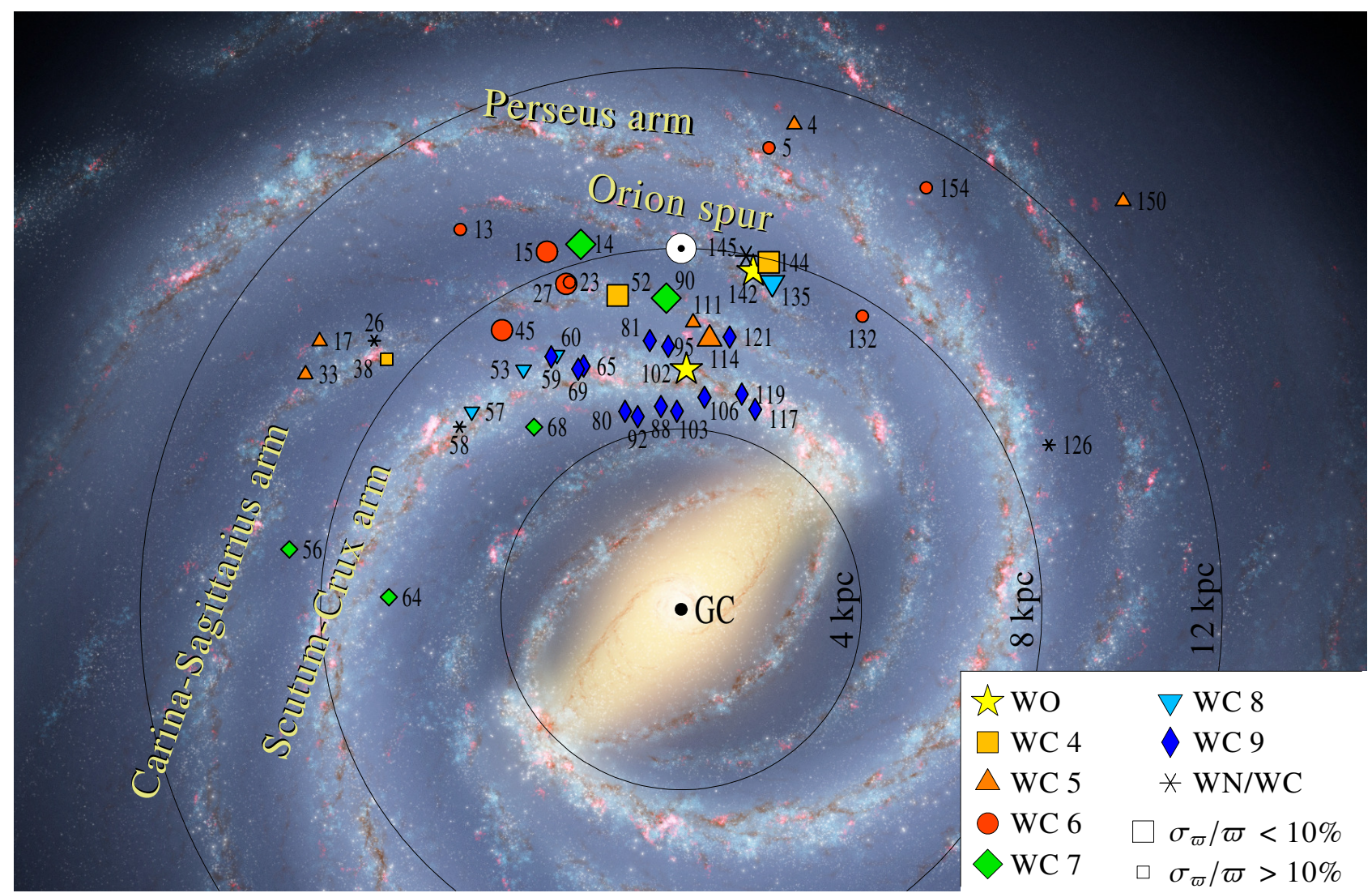

Fig. 1. Positions in the Galactic plane of the Galactic WC and WO sample with the positions of the sun ( $\odot)$ and the Galactic Center (GC) indicated. The different symbols depict the various subtypes and larger symbols denote lower parallax errors as explained in Fig. 3. For illustration purposes, an artist's impression of the Milky Way has been put in the background with certain spiral arms highlighted. Background image credit: NASA/JPL-Caltech/ESO/R. Hurt

a Rosseland continuum optical depth of $\tau=20 . \dot{M}$ denotes the mass-loss rate, $v_{\infty}$ the terminal wind velocity and $D$ is the clumping factor as introduced in Hamann \& Koesterke (1998). Using the definition of the distance modulus

D.M. $=5 \log \frac{d}{10 \mathrm{pc}}$,

we can compare the new distances directly to those applied in Sander et al. (2012). With the help of the Stefan-Boltzmann equation

$L=4 \pi R_{*}^{2} \sigma_{\mathrm{SB}} T_{*}^{4}$,

connecting $R_{*}$ and $T_{*}$ to the stellar luminosity $L$, as well as Eq. (1), we can immediately obtain

$\Delta \log L=0.4 \cdot \Delta \mathrm{D} . \mathrm{M}$.

$\Delta \log R_{*}=0.5 \cdot \Delta \log L$

$\Delta \log \dot{M}=0.75 \cdot \Delta \log L$

with $\Delta$ denoting the difference between the new values based on the Gaia parallaxes and the old values from the 2012 paper.

Utilizing the new distances and the Galactic coordinates, we can plot the locations of the sample WC and WO stars projected on the Galactic plane as depicted in Fig. 1. Though the background is an artist's impression, the spiral arm structure is roughly based on our current knowledge of the Milky Way structure. As expected, most WC stars and both WO stars are located in the spiral arms. For a more in-depth discussion about the spatial distribution of the Galactic WR stars, although not yet based on the Gaia DR2 results, we refer to Rosslowe \& Crowther (2015).

\section{Results}

Applying the methods and equations outlined above, we obtain the new distances as well as the dependent quantities, such as luminosities and mass-loss rates. After reviewing the errors, this section presents the results and puts them into context, checks the validity of an $M_{v}$ calibration per subtype, and discusses noteworthy outcomes for a few particular objects.

\subsection{Error estimates}

In our previous analysis of the Galactic WC stars (Sander et al. 2012), the spectroscopic parameters $\left(T_{*}, R_{\mathrm{t}}\right)$ were determined by assigning the best model from a grid of WC atmospheres reproducing the normalized line spectrum of the available observations. The uncertainties of the assigned spectroscopic parameters roughly correspond to the span between neighboring models in the grid. For dense winds, there is a certain parameter degeneracy (Hamann et al. 2003). The luminosities were derived from reproducing the spectral energy distribution (SED), which requires an input of the reddening parameter $E_{b-v}$ and the distance. While the former could be determined quite accurately with an uncertainty of about $\pm 0.02 \mathrm{mag}$, the latter depended on the often uncertain membership of the particular object to a cluster or association and the uncertainty of their distance themselves. Thus, the distance moduli had an estimated error 
margin of about $\pm 0.75 \mathrm{mag}$, outweighing all other uncertainties that enter the luminosity estimate. In this work, we focus only on the distances and their uncertainty. As mentioned in Sect. 2, the distances calculated from the Gaia DR2 parallaxes by Bailer-Jones et al. (2018) come with uncertainty estimates intrinsic to their Bayesian method, defined by the the lower and upper boundaries of an asymmetric confidence interval that corresponds to one standard deviation in the case of the distance distribution being described by a Gaussian. With these uncertainties given, we calculate the resulting uncertainties for the distance modulus, the luminosity and the mass-loss rate according to the Eqs. (2-6). We are aware that these would also propagate into the new values of $R_{*}$, but this value listed in Table 1 serves more as a reference quantity between different types of WC stars.

We calculate the stellar masses by inverting Eq. (19) from Langer (1989) that connects masses and luminosities of WC stars. Unlike hydrogen-free WN stars, which should be from a theoretical standpoint relatively close to ideal He main sequence stars, WC stars already have a significant carbon and oxygen fraction at the surface. Consequently, helium is depleted and unless the star is fully mixed - even more so in the inner layers down to the core. Therefore, the helium (surface) mass fraction enters this relation as an additional parameter, although at a moderate degree. As an example, for a WC star with $\log L / L_{\odot}=5.8$, the masses range from $21.7 M_{\odot}$ in the case of no helium at all to $24.1 M_{\odot}$ in the case of pure helium, which would of course technically no longer be classified as a WC star. Assuming that the helium mass fraction lies in a realistic range of $X_{\mathrm{He}}=0.3 \ldots 0.8$, the previous example would lead to an uncertainty of $\sim 1 M_{\odot}$. This is much lower compared to the uncertainty in the luminosity and thus is ignored in our error estimate given in Table 1.

To check whether different WC masses would be derived from newer stellar evolution models with e.g. updated compositions, opacities, or nuclear energy generation rates, we compare the relation from Langer (1989) with the location of selected stages ("snapshots") from different stellar evolution codes in the massluminosity plane. This is depicted in Fig. 2, where we plot the snapshot of evolution models with carbon surface mass fractions $X_{\mathrm{C}}=0.4$ for the Geneva (Ekström et al. 2012) and FRANEC (Chieffi \& Limongi 2013) codes which account for rotation, as well as single star models calculated with the STARS code in 2010 from BPASS (Eldridge \& Stanway 2009). For comparison, also the relation for chemically-homogeneous hydrogen-free WN stars from Gräfener et al. (2011) is shown. Since some tracks do not reach $X_{\mathrm{C}}=0.4$, which is the standard carbon abundance in our sample, we also show selected snapshots of $X_{\mathrm{C}}=0.17$ where a star should already have a WC-type spectrum (assuming of course a sufficiently high mass-loss rate). While the relation from Langer (1989) used in this work gives masses that are within $1 M_{\odot}$ of the He-star relation, the evolution models yield a bit lower masses, typically by $1-2 M_{\odot}$ for the models resulting from the STARS and Geneva code. The FRANEC results differ a bit more from the other models, especially for the lower masses where the models do only reach $X_{\mathrm{C}}=0.17$. Since these values are just based on snapshots and none of the papers provide a mass-luminosity relation for WC stars, we stick with the relation from Langer (1989), but point out that the WC masses derived here could be slightly overestimated.

\subsection{Luminosities, temperatures and HRD positions}

Applying the new luminosities based on Gaia DR2 parallaxes and taking $T_{*}$ from Sander et al. (2012), we obtain the revised positions of the Galactic WC and WO stars in the Hertzsprung-

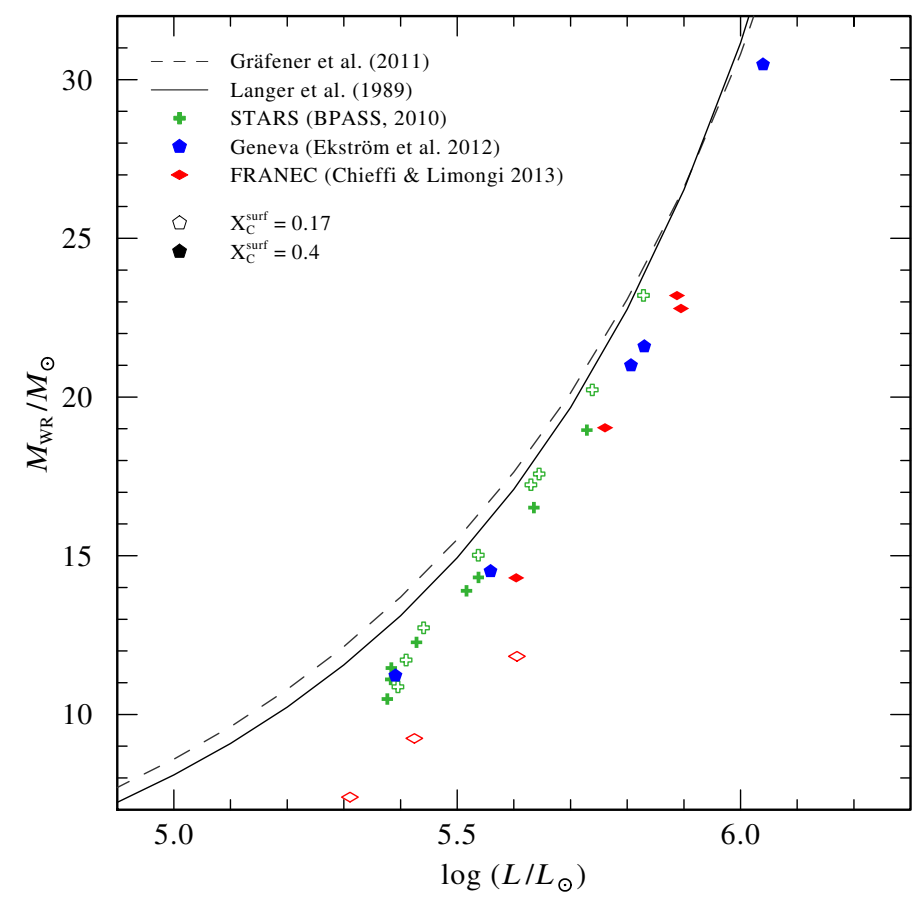

Fig. 2. Comparison of the mass-luminosity relations from Langer (1989) for WC stars and Gräfener et al. (2011) for hydrogen-free WN stars with snapshots of different stellar evolution models showing Carbon surface abundances which are commonly attributed to a WC spectral appearance.

Russell diagram (HRD) as shown in Fig. 3. For comparison, the HRD with the luminosities used in the 2012 paper is shown in Fig. 4. While a number of stars more or less remain at their previous position, most of them change, some even quite drastically. Already at first sight it becomes clear that the luminosity range between $\log L / L_{\odot}=5.6$ and 6.0, which was only populated by the two WO2 stars previously, is now also covered by various stars from the subtypes WC5 to WC9. This previous upper luminosity limit was likely too low due to the $M_{v}$ calibration as we discuss in Sect. 3.4.

With the exception of the WC4 stars, all other WC subtypes cover a broad range of luminosities. This is in particular true for the WC9 subtype, ranging over more than one order of magnitude in $L$ instead of just clustering around $\log L / L_{\odot} \approx 5.2$ in the 2012 paper. The reason for the earlier results was the fact that only one star, WR 95, had a constrained distance and thus all other WC9 stars were calibrated to its magnitude. While the results based on Gaia DR2 confirm the HRD position of WR 95, they also reveal that there is a significant spread in luminosities for WC9 stars, independent of whether or not they are WC9d, that is showing infrared dust excess. As we discuss in Sect. 3.5, the two most luminous WC9 stars are most likely binaries, which means that their luminosity in Table 1 would be overestimated. However, even with a significant luminosity reduction of these two stars, for example by $0.3 \mathrm{dex}$, the spread in WC9 luminosities would still be about one order of magnitude.

Apart from the change in the overall picture due to the revised distance, there are several noteworthy objects that significantly altered their obtained luminosities. These is discussed in Sect. 3.5.

The temperatures of the WC stars have been a constant topic of discussion in the past years. Since one can safely assume that WC stars are core-helium burning objects and do not have a hydrogen layer, one would expect them to appear relatively 


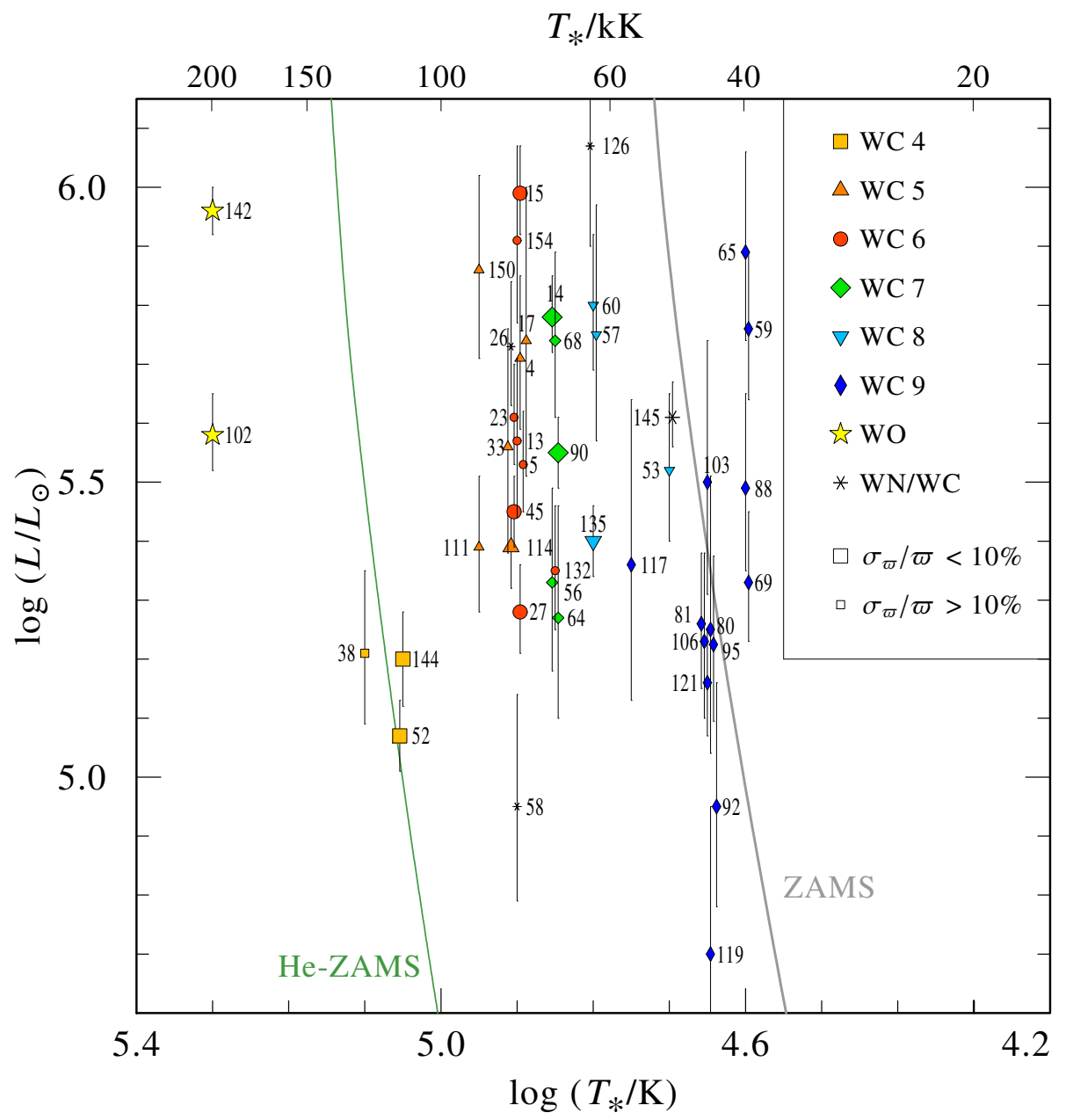

Fig. 3. Hertzsprung-Russell diagram of the Galactic WC \& WO stars with updated luminosities due to Gaia distances: The sample is taken from Sander et al. (2012), reduced to those with Gaia parallaxes that have a relative error of less then $40 \%$. The vertical bars denote the error in luminosity resulting from the distance uncertainty. Tiny horizontal shifts have been applied to different different symbols for clarity. Stars with parallax errors of less than ten percent are represented by larger symbols. The zero age main sequence (ZAMS, from Ekström et al. 2012, here for stars without initial rotation) and for pure helium stars (HeZAMS, from Langer 1989) are depicted as gray and green solid lines. close to the zero age main sequence (ZAMS) for pure helium stars (He-ZAMS) or even hotter than that as they come relatively close to the theoretical concept of an evolved helium star. However, Fig. 4 shows that this is only the case for the WC4 and WO stars, while all other subtypes show much cooler temperatures. To properly put this into perspective, one has to be aware of the different temperatures that are referred to in the literature. The primary input parameter of a PoWR model is the temperature $T_{*}$, which is defined as the effective temperature according to the Stefan-Boltzmann Eq. (3) at a Rosseland continuum optical depth of $\tau=20$. For OB stars and stars of later spectral types, an effective temperature is often defined at a much lower optical depth, namely at $\tau=2 / 3$, which we denote as $T_{2 / 3}$ here to avoid any confusion. For stars with dense winds such as the Galactic WR stars, the difference between $T_{2 / 3}$ and $T_{*}$ can be up to a factor of two or even more in extreme cases with $T_{2 / 3}$ consequently being much smaller than $T_{*}$. In particular, models with different $T_{*}$ can have the same $T_{2 / 3}$ (see, e.g., Fig. 4 in Todt et al. 2015), illustrating the parameter degeneracy mentioned in Sect. 3.1. In the dense wind regime, models with the same $T_{2 / 3}$ tend to have relatively similar spectra, meaning that to a certain degree one could shift them to a different, i.e. also higher, $T_{*}$ (cf. Sect. 4 in Hamann \& Gräfener 2004). Nevertheless, tests have shown that it is not possible to obtain a satisfactory fit for all stars by shifting them to the high temperatures of the He-ZAMS as the models yield too strong emission lines, but more studies on this topic are currently underway. The unanswered questions regarding optically thick winds of WR stars and their consequences for the mass-loss are a major topic of current research, as demon- strated by several recent publications (e.g. Gräfener et al. 2017; Nakauchi \& Saio 2018; Grassitelli et al. 2018).

Aside from the modeling aspect, one also has to be aware of the temperature definition used to construct the He-ZAMS. In our figures, we plot the He-ZAMS from Langer (1989), but the choice of the set of evolutionary models is of no particular importance since all of them provide a temperature corresponding to the hydrostatic layers, not corrected for any wind. Some evolutionary models try to account for the wind layers and thus also provide a "corrected" temperature that could be compared to $T_{2 / 3}$, but this correction is usually done in a rather approximative way. So far, only a pilot study by Groh et al. (2014) exists where detailed atmosphere models were calculated for various points of the evolutionary tracks, also demonstrating that more approximate corrections seem to be way off for Wolf-Rayet stars. Comparing the "uncorrected" temperatures with $T_{*}$ is therefore still usually the best method. This works well if the atmosphere models have a (quasi-)hydrostatic stratification at the definition radius of $T_{*}$, i.e. $\tau=20$. For $\mathrm{OB}$ star models this is always the case, but for Wolf-Rayet stars this is not guaranteed by default. Especially models with dense winds can have $v\left(R_{*}\right) \gg 1 \mathrm{~km} \mathrm{~s}^{-1}$, meaning that they would not at all be hydrostatic even in these deep layers. Fortunately, the models applied in Sander et al. (2012) mostly have $v\left(R_{*}\right)=$ $0.1 \ldots 1 \mathrm{~km} \mathrm{~s}^{-1}$, and thus this does not seem to be an issue here, at least as long as we assume that a revision of the stellar atmosphere models would not lead to a major change. This assumption seems justified from our experiences with particular sources and the mostly decent changes with regards to the spectral 


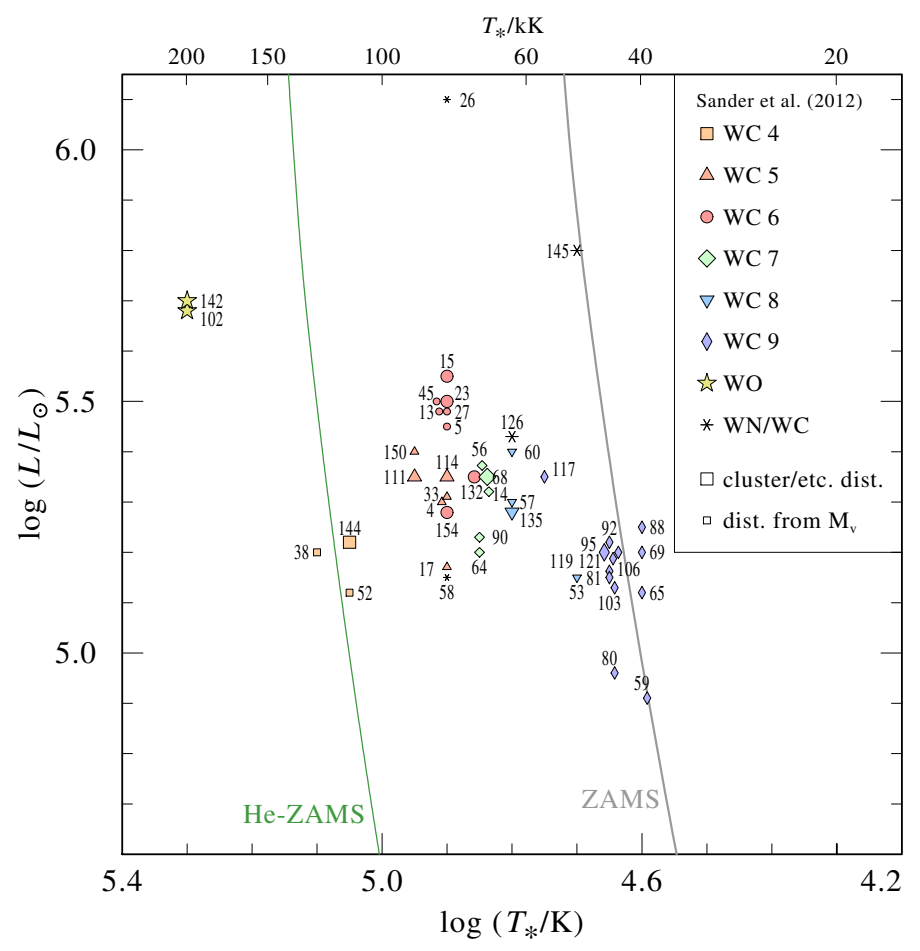

Fig. 4. Hertzsprung-Russell diagram of the Galactic WC \& WO stars with their luminosities as in the Sander et al. (2012) paper. The larger symbols denote stars where a direct information for the distance, e.g. from cluster membership, was known. The smaller symbols denote stars with luminosities that were obtained via a subtype-magnitude calibration.

appearance when we updated our model grids with the latest code version.

If the temperature discrepancy cannot be explained by parameter degeneracy or different physical conditions that would prevent a proper comparison, it must reflect a more fundamental issue. Since for a given luminosity, the temperature $T_{*}$ directly corresponds to a radius $R_{*}$ via the Stefan-Boltzmann relation, this has also been termed the "WR radius problem". Pointing out this problem and building on ideas by Ishii et al. (1999) and Petrovic et al. (2006), Gräfener et al. (2012a) demonstrated that by accounting for reasonable clumping factors in the observed range, there could be an inflated envelope that would essentially lead to a bending of the He-ZAMS toward lower temperatures. This inflation is caused by the proximity to the Eddington limit $\left(\Gamma_{\mathrm{e}}>0.3\right)$ and only occurs when the outer boundary temperature for the stellar structure model, which should roughly correspond to the temperature at the sonic point, is below $\approx 70 \mathrm{kK}$. Considering this limit could explain why the subtypes later than WC6 are inflated while the WC4 class is not. The WC5 and WC6 stars have stellar temperatures between 70 and $100 \mathrm{kK}$, which would be a challenge to this picture as they seem to be inflated allthough they should not be according to Gräfener et al. (2012a), but this discrepancy might be arbitrary as the sonic point of the models used in Sander et al. (2012) is not really well defined due to the use of a fixed velocity stratification with $\beta=1$, which is most likely not valid in the deeper layers. Moreover, the WC5 star WR111 was modeled by Gräfener \& Hamann (2005) with a hydrodynamically consistent model using $T_{*}=140 \mathrm{kK}$, thus pointing at the possibility that with a more adequate stratification also WC5 and maybe even WC6 stars might be explained by models that do not require an inflation at all. With the atmosphere models and the luminosities given, we can obtain the val- ues for $\Gamma_{\mathrm{e}}$ using the mass-luminosity relation for WC stars from Langer (1989). For our sample stars, the derived values for $\Gamma_{\mathrm{e}}$ are between 0.2 and 0.4 for most of the stars with an average of $\Gamma_{\mathrm{e}}=0.3$ for all subtypes from WC5 to WC8, while the WC9 stars have a mean of 0.23 with a large scatter between 0.11 (WR 119 ) and 0.38 (WR 65). The likely non-inflated WC and WO stars have with $\Gamma_{\mathrm{e}}^{\mathrm{WC} 4} \approx 0.2$ and $\Gamma_{\mathrm{e}}^{\mathrm{WO}} \approx 0.4$ very different mean values. Taking these values, not all sample stars would fulfill the necessary inflation condition from Gräfener et al. (2012a), but we view this only as a minor concern as the we did not perform an in-depth atmosphere analysis for each of the stars in Sander et al. (2012) and relied on grid models for most objects. With the resulting uncertainty in $\Gamma_{\mathrm{e}}$, most of the sample stars with subtypes later than WC4 are thus likely to fulfill the condition of $\Gamma_{\mathrm{e}}>0.3$.

The inflation of Wolf-Rayet radii was also deduced by Glatzel (2008) on the basis of numerical simulations of strangemode instabilities evolving into the nonlinear regime for a WolfRayet star model, which was motivated by the hydrogen-free WN star WR 123. Glatzel (2008) concluded that the star inflates due to consecutive shock waves generated by strange-mode instabilities, which would push the matter to larger radii and potentially even launch the stellar wind. While the latter statement would be a huge paradigm shift and might not be the full truth given that Gräfener \& Hamann $(2005,2008)$ demonstrated that radiative driving plays an important role, there is a certain level of consistency between Glatzel (2008) and Gräfener et al. (2012a) as well as Gräfener \& Vink (2013) about where inflation starts and the restriction to certain opacity regimes. We come back to this issue when discussing stellar evolution in Sect. 4.

\subsection{Mass-loss rates}

With the revised luminosities being given and adopting the distance-independent parameters $\left(T_{*}, R_{\mathrm{t}}, v_{\infty}, D\right)$ from Sander et al. (2012), we obtain the revised mass-loss rates via Eq. (6). The results are compiled in Table 1 including the uncertainties as discussed in Sect. 3.1. With now both $L$ and $\dot{M}$ given, we can check how the relation between mass-loss and luminosity is affected and how our results compare to the relation from Nugis \& Lamers (2000), which is widely applied in stellar evolution calculations. Figure 5 shows the revised results. While the slope of linear regression fit in Sander et al. (2012; 0.83) was in line with Nugis \& Lamers (2000) obtaining 0.84, the updated figure suggests that the luminosity-dependence is weaker, yielding

$\log \frac{\dot{M}}{M_{\odot} \mathrm{yr}^{-1}}=-8.31( \pm 0.29)+0.68( \pm 0.05) \cdot \log \frac{L}{L_{\odot}}$,

with the given errors denoting the formal standard deviations obtained by the linear regression. For comparison, we also show the results for the LMC WC stars analyzed by Crowther et al. (2002) in Fig. 5. They tend to pretty much blend into the cloud of Galactic data points, while a closer inspection shows that three of the six objects, namely those with slightly lower mass-loss rates (BAT99 8, BAR999, and BAT99 90) seem to match the relation of Nugis \& Lamers (2000) assuming a carbon mass fraction of 0.6. However, this is a pure coincidence as neither the different iron abundance - Crowther et al. (2002) used $0.4 Z_{\odot}$ for his LMC models - is taken into account, nor does the carbon fraction match. Only one of them (BAT99 8) has $X_{\mathrm{C}} \approx 0.5$ while the other two have a value close to or even below $X_{\mathrm{C}}=0.4$. Due to the two groups, the $\dot{M}-L$-relation for the LMC WC4 stars is much steeper, having a slope of 1.29. 


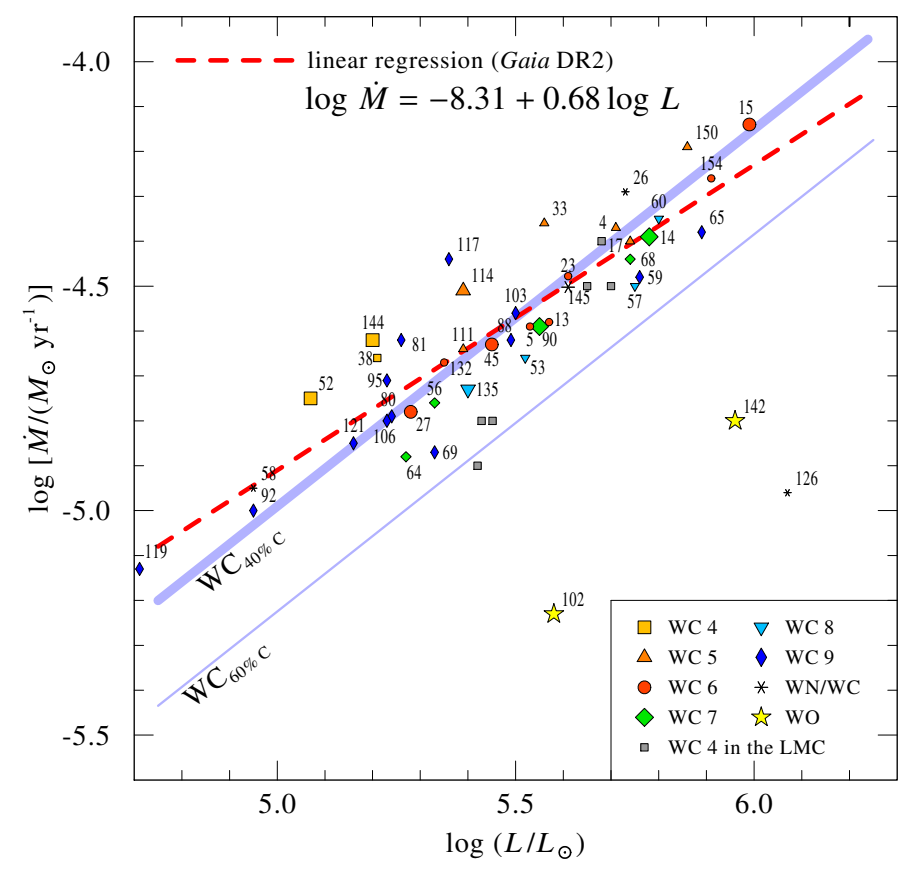

Fig. 5. Empirical mass-loss rates versus updated luminosity for the Galactic WC \& WO stars. The red-dashed line gives the linear regression fit to these results. The relations proposed by Nugis \& Lamers (2000) for WC stars with $40 \%$ and $60 \%$ carbon, respectively, are indicated by the thick and thin shaded lines. The results from Crowther et al. (2002) for the LMC WC4 stars are denoted by gray symbols.

The fact that the LMC WC4 stars are at higher luminosities compared to their Galactic counterparts is expected due to the different metallicity $Z$. Nevertheless, it should be noted that this comparison might be slightly biased due to the fact that the models used by Crowther et al. (2002) do not contain ions higher than Fe VIII. Since we see for the Galactic results that especially for early WC subtypes the inclusion of high Fe ions is crucial, this might explain why the LMC WC4 stars are reproduced with cooler models. Due to the parameter degeneracy for dense winds, such a cooler "solution" in terms of spectral appearance is often easy to find, but unfavorable or even unphysical from a hydrodynamical standpoint as demonstrated by Gräfener \& Hamann (2005).

As discussed in length in Sander et al. (2012), the finding that most of the models align in the $\log R_{\mathrm{t}}-\log T_{*}$-plane, would imply a slope of 0.75 in the case of the terminal velocity $v_{\infty}$ (and clumping factor $D$ ) being the same for all stars. Interestingly, the new distances now lead to a flatter slope, while the value in Sander et al. (2012) was steeper. We can try to eliminate the effect of $v_{\infty}$ by including an explicit velocity dependence in the relation, leading to

$$
\begin{aligned}
\log \frac{\dot{M}}{M_{\odot} \mathrm{yr}^{-1}}= & -9.47( \pm 0.30)+0.62( \pm 0.04) \cdot \log \frac{L}{L_{\odot}} \\
& +0.44( \pm 0.08) \cdot \log \frac{v_{\infty}}{\mathrm{km} \mathrm{s}^{-1}}
\end{aligned}
$$

which actually flattens the slope even further.

To obtain a relation similar to Nugis \& Lamers (2000) we can also account for the helium abundance, which is slightly different in some of the sample stars. Due to $Z=1-Y$ for hydrogenfree stars, explicitly accounting for both terms is not useful and we simply obtain

$\log \frac{\dot{M}}{M_{\odot} \mathrm{yr}^{-1}}=-8.68( \pm 0.31)+0.71( \pm 0.04) \cdot \log \frac{L}{L_{\odot}}$

$$
-0.74( \pm 0.29) \cdot \log X_{\mathrm{He}}
$$

While there is indeed a slight steepening in the luminosity dependence, this has to be taken with care as only very few sample stars in Sander et al. (2012) actually used models with nongrid abundances. Moreover, Tramper et al. (2016) suggested to introduce a term depending on $X_{\mathrm{Fe}}$, but since all models in Sander et al. (2012) use the abundance of $X_{\mathrm{Fe}}=1.6 \times 10^{-3}$, this is not an option inside this sample. While from a theoretical point of view $X_{\mathrm{Fe}}$ is an excellent parameter for a mass-loss relation, as its slope would characterize one of the most important wind driving contributors, the actual determination of $X_{\mathrm{Fe}}$ in an observation is tough and usually requires excellent UV spectra resolving features of the iron forest which then also has to be precisely reproduced by the atmosphere model. Presently, very few attempts for such work exists (e.g. Herald et al. 2001) and the assumption of a "typical" iron abundance motivated by the host galaxy is standard, not only when analyzing WR stars, but also other hot, massive stars. For WN stars, Hainich et al. (2015) determined the dependence of $\dot{M}$ on $X_{\mathrm{Fe}}$ by studying the WN populations of different galaxies with different metallicities. This is an excellent approach that holds as long as the spread in $X_{\mathrm{Fe}}$ inside the WN population of a particular galaxy is small, but as mentioned before this is unfortunately tough to constrain from observations and would require more and better UV spectroscopy, highlighting the urgent demand for a new UV telescope filling the oncoming gap after the end of the HST.

From an observational point of view, assuming a negligible iron abundance spread inside the LMC, a glimpse of a potential $X_{\mathrm{Fe}}$-dependency of the WC mass-loss rates can be obtained from comparing our results with those by Crowther et al. (2002). Adding further points at other metallicities turns out to be tough. In the SMC, no WC stars are known. There is one WO (SMC AB 8) which is part of a binary system analyzed by Shenar et al. (2016). However, mixing WCs and WOs here would be a problem as we know, e.g., from Fig. 5, that the mass-loss rates of the WO stars are lower than those of WCs with the same luminosity. A comparison of only the WO stars would in principle be an option, but apart from the low number of known objects the question would remain whether a result could also be applicable to the larger WC population.

Instead we use the fact that all known WC stars in the LMC have a similar spectral type (WC4) and compare them to their Galactic counterparts. Two additional data points are obtained from Abbott et al. (2003) and Abbott (2004), who analyzed several WR stars in different galaxies, including two WC4-5 stars ([MC83] 70 alias MC70, and [MC83] 79 alias MC79) in the outskirts of M 33, where the metallicity is SMC-like. To eliminate the luminosity-dependence, we use the "transformed mass-loss rate"

$\dot{M}_{\mathrm{t}}=\dot{M} \sqrt{D} \cdot\left(\frac{1000 \mathrm{~km} \mathrm{~s}^{-1}}{v_{\infty}}\right)\left(\frac{L}{10^{6} L_{\odot}}\right)^{3 / 4}$,

introduced by Gräfener \& Vink (2013). The value of $M_{\mathrm{t}}$ can be understood as the mass-loss rate $\dot{M}$ the star would have, if it had a smooth wind (i.e., $D=1$ ), a terminal wind velocity of $1000 \mathrm{~km} \mathrm{~s}^{-1}$ and - most importantly here - a luminosity of $10^{6} L_{\odot}$. Since the Galactic sample of WC4 stars consists of three stars, we also considered the WC5 stars, which essentially have the same $M_{\mathrm{t}}$ as the WC4 stars. This is a consequence of Eq. (5) in Sander et al. (2012), leading to all WC4 to WC7 stars in our Galactic sample having $\log \dot{M}_{\mathrm{t}} \approx-4.06$. For the LMC sample with their more tailored models, the scatter in $\log \dot{M}_{\mathrm{t}}$ is larger. Nonetheless, for both samples as well as MC70, the calculation 


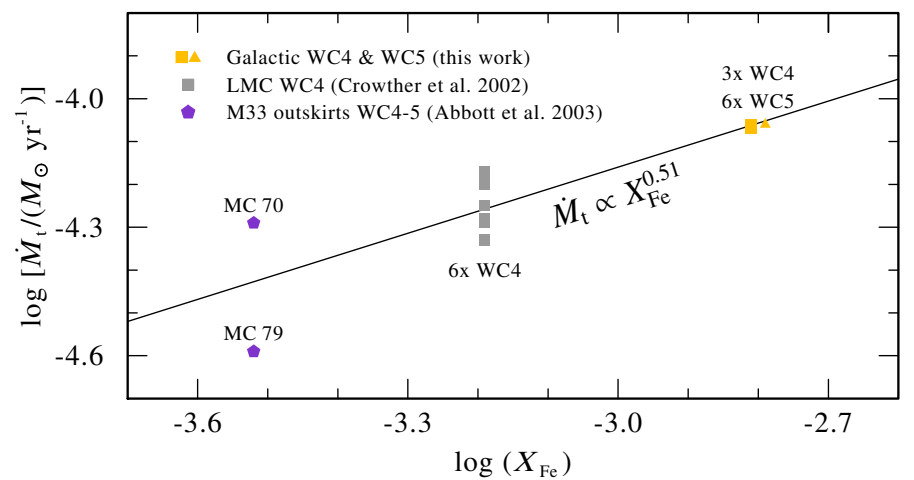

Fig. 6. Transformed mass-loss rate versus Fe mass fraction for WC4 and WC5 stars at different metallicities: The extragalactic results are taken from Crowther et al. (2002, LMC) and Abbott et al. (2003; M33 outskirts). The solid line gives the linear regression fit to the data points. The Galactic WC4 and WC5 plot symbols have a slight shift compared to the actual datapoint to ensure visibility.

of $M_{\mathrm{t}}$ is straight forward, but we had to assume $v$ for MC79 and choose a typical value for a WC4 of $v_{\infty}=3000 \mathrm{~km} \mathrm{~s}^{-1}$.

The resulting distribution of $\dot{M}_{\mathrm{t}}$ over $X_{\mathrm{Fe}}$ can be seen in Fig. 6, where we also show a linear fit to the double-logarithmic data. Including the two sources in M 33, we obtain

$\log \dot{M}_{\mathrm{t}}=0.51( \pm 0.06) \cdot \log X_{\mathrm{Fe}}-2.62( \pm 0.19)$.

A dependency of approximately $X_{\mathrm{Fe}}^{0.5}$ for WC stars is in line with the suggestion from Crowther et al. (2002), but significantly more flat than what was found for WN stars by Hainich et al. (2015). And even though the data points for M 33 in Fig. 6 might have a considerable uncertainty, this result would essentially not change when leaving them out, as we then obtain $X_{\mathrm{Fe}}^{0.49}$ instead. With Eq. (10) our relation thus points at a dependence for the true mass-loss rate of approximately

$\dot{M} \propto D^{-1 / 2} v_{\infty} L^{3 / 4} X_{\mathrm{Fe}}^{0.5}$

From a theoretical point, the issue of metallicity-dependent mass-loss rates for WC stars has been studied by Vink \& de Koter (2005). While they do not give an explicit $X_{\mathrm{Fe}^{-}}$ dependence, their $Z$-definition does not include the self-enriched carbon and oxygen in the WCs. Thus their $\dot{M}(Z)$-slope reflects mainly the change of the primary wind driving contributor, which is iron. In total they studied regimes from $10 Z_{\odot}$ down to $10^{-5} Z_{\odot}$ with a slope of 0.66 in the region. This is slightly steeper than the 0.5 we obtain here, but given the scatter for the LMC stars and the uncertainty of the M33 data points, still in agreement with what we obtain from the observations.

The well used recipes of Nugis \& Lamers (2000) do not provide an explicit term for the Fe-dependence, but instead just use a $Z$-term containing all elements beyond He. Of course the two main contributions to $Z$ in WC stars are carbon and oxygen. We made a few test calculations to study the impact of abundance changes on the mass-loss rate using the work ratio

$Q:=\frac{\dot{M} \int\left(a_{\mathrm{rad}}-\frac{1}{\rho} \frac{\mathrm{d} P}{\mathrm{~d} r}\right) \mathrm{d} r}{\dot{M} \int\left(v \frac{\mathrm{d} v}{\mathrm{~d} r}+\frac{G M_{*}}{r^{2}}\right) \mathrm{d} r}$,

which is a proxy for the global hydrodynamic consistency of the model. In a nutshell, a change in $Q$ between models that are identical in everything but different abundances means that the mass-loss rate should be affected. More information about

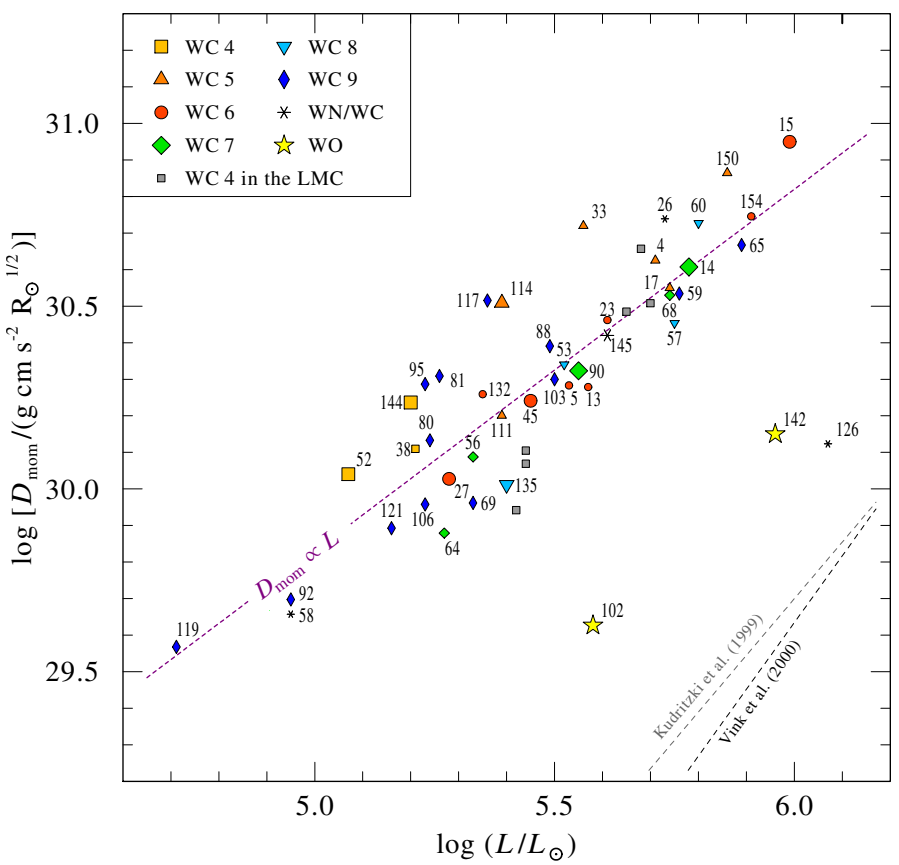

Fig. 7. Modified wind momentum versus updated luminosity for the Galactic WC \& WO stars. The purple-dashed line gives the linear regression fit to these results. For comparison, the relations for OB stars from Kudritzki et al. (1999) and Vink et al. (2000) are shown as gray and black dashed lines. The gray symbols without labels denote the results from Crowther et al. (2002) for the LMC WC4 stars.

the concept of the work ratio can for example be found in Gräfener \& Hamann (2005), Sander et al. (2017, 2018). Our test calculations for a WC5 grid model show that neither a variance of $\mathrm{C}$, nor of $\mathrm{O}$, do really impact the work ratio, at least not if the variations are in the observed range of abundances for WC stars, i.e. between 0.2 and 0.6 for $X_{\mathrm{C}}$ and 0.05 and 0.15 for $X_{\mathrm{O}}$. Of course a proper quantification of the $\dot{M}$-dependence on $\mathrm{C}$ and O would be preferable. Vink \& de Koter (2005) did this with a Monte Carlo approach, but a CMF-based calculation with a locally consistent acceleration similar to what has been done for hydrogen-containing WN stars by Gräfener \& Hamann (2008) does not yet exist. However, this is way beyond the scope of the present paper and we thus will come back to this in future studies. The concept of the work ratio has also been recently applied to obtain mass-loss rates from grids of models for the bi-stability domain in a study by Petrov et al. (2016). In this work we only used it as a first step to ensure that the impact is small.

Our finding does not imply that carbon and oxygen are not contributing to the wind driving. However, our test calculations hint that their precise abundances do not really alter the resulting mass-loss rates, at least at Galactic metallicites. Thus, a massloss recipe with a simple $Z$-dependence is not properly reflecting the physical dependencies of $\dot{M}\left(X_{\mathrm{Fe}}\right)$. These recipes - including the ones from Nugis \& Lamers (2000) and also our own ones given in Eqs. (7-9) - are best representations to a certain dataset, but their validity is limited to the parameter range they have been established in. Thus, extrapolations of these formulae into different regimes, for example lower masses or different metallicities, are dangerous, and a proper treatment would require a more physically motivated parametrization.

Instead of plotting just the mass-loss rate, we can also show the modified wind momentum $D_{\text {mom }}=\dot{M} v_{\infty} \sqrt{R_{*}}$ (e.g. Kudritzki et al. 1999) versus the luminosity as displayed in Fig. 7. This quantity is motivated by the analytical calculations 
from Kudritzki et al. (1989) based on extensions of the CAK (named after Castor et al. 1975) theory for line-driven winds, yielding

$\dot{M} v_{\infty} \sqrt{R_{*}} \propto \hat{k}^{\frac{1}{\alpha-\delta}} L^{\frac{1}{\alpha-\delta}}$,

or simply $D_{\text {mom }} \propto L^{1 / \alpha_{\text {eff }}}$ with $\alpha_{\text {eff }}:=\alpha-\delta$ and $\hat{k}, \alpha$, and $\delta$ denoting the so-called "force multiplier parameters". A brief summary of the concept behind this relation is for example given in Kudritzki et al. (1998). This means that not only does the modified CAK (mCAK) theory predict a tight relation between $D_{\text {mom }}$ and $L$, but also that the reciprocal value of the exponent should reflect the force multiplier parameter $\alpha$ describing the line strength distribution function. Kudritzki et al. (1999) found based on studies of relatively nearby stars - that $\alpha_{\text {eff }}$ seems to increase from A-type to early B-type stars and then mildly decreases to a value of $\alpha_{\mathrm{eff}} \approx 0.65$ for O-type stars. A linear relation fit of our revised WC results now yields

$$
\log \frac{D_{\mathrm{mom}}}{\mathrm{g} \mathrm{cm} \mathrm{s}^{-2} R_{\odot}^{1 / 2}}=24.88( \pm 0.44)+0.99( \pm 0.08) \cdot \log \frac{L}{L_{\odot}}
$$

meaning that essentially $D_{\text {mom }} \propto L$ and $\alpha_{\text {eff }} \approx 1$. This would imply a further flattening of the exponent $1 / \alpha_{\text {eff }}$ compared to OB stars, something also found for the WN stars in the LMC by Hainich et al. (2015). Interestingly, the same exponent of $\approx 1$ was also found for the hydrogen-poor WNL stars in M31 (Sander et al. 2014). These results are in conflict with the numerical calculation of $\alpha_{\text {eff }} \approx 0$ by Gräfener \& Hamann (2005) for their hydrodynamically consistent WC star model. This illustrates that our linear relation between $D_{\text {mom }}$ and $L$ is only an empirical result and one should be careful when interpreting this in the scheme of the mCAK. As for example reviewed in Puls et al. (2008), an mCAK parametrization does not hold for optically thick winds with massive line overlap, which is essentially the situation we have for Galactic WR winds. The necessity to account for multiline scattering when describing the radiative driving of the wind is also reflected in the values obtained for the wind efficiency parameter

$\eta:=\frac{\dot{M} v_{\infty} c}{L}$,

listed in the last column of Table 1. With the exception of the also otherwise odd transition-type star WR 126 (see discussion in Sect. 3.5), all objects in our sample significantly exceed the single-scattering limit of $\eta=1$. For several objects, especially those of early WC subtypes, $\eta$ even exceeds values of 10 or 20. It is challenging to explain these high values with a purely radiatively driven wind, even when properly accounting for multiple scattering. To shed more light on these issue, more hydrodynamically consistent models (e.g. Gräfener \& Hamann 2005, 2008 ; Sander et al. 2017) covering the dense wind regime are needed.

\subsection{Subtype-magnitude relation}

With the distances now given, we can check the subtypemagnitude relation used in earlier works. In the past, with only few Galactic Wolf-Rayet stars having a suggested distance, for example from cluster memberships, it was common to assume that the absolute magnitude

$M_{v}=v-$ D.M. $-A_{v}$

in the narrowband system from Smith (1968b) is approximately the same within one Wolf-Rayet subtype. As long as a few or

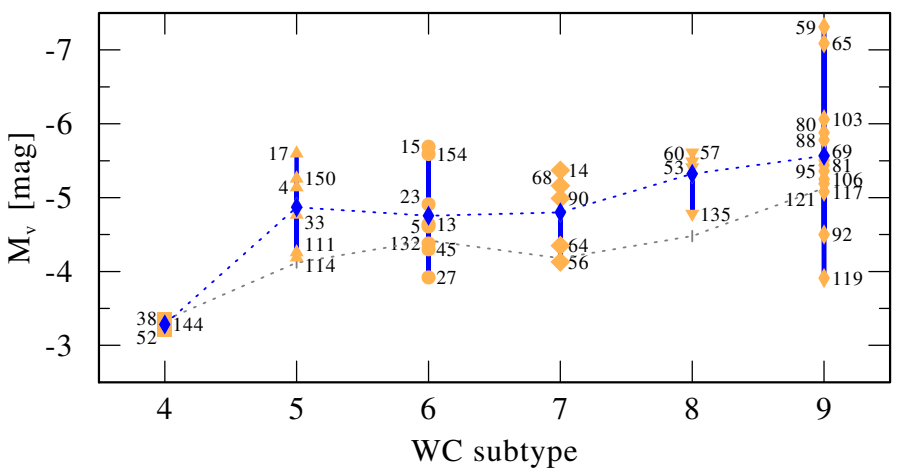

Fig. 8. Mean absolute magnitudes of the WC stars in the Milky Way depicted by subtype: The orange symbols indicate the positions of the individual stars with the thick blue lines marking the span in magnitudes. The blue diamonds connected by the dotted blue line mark the mean value per subtype. The gray dotted line denotes the calibration result from Sander et al. (2012).

Table 2. Mean absolute magnitudes for the different WC subtypes derived from the revised results based on Gaia DR2 (cf. Fig. 8) compared to the calibration from Sander et al. $\left(2012 ; M_{v}^{2012}\right)$ using stars with distances known from their cluster or association membership.

\begin{tabular}{lcc}
\hline \hline Subtype & $M_{v}(\mathrm{mag})$ & $M_{v}^{2012}(\mathrm{mag})$ \\
\hline WC4 & -3.28 & -3.34 \\
WC5 & -4.87 & -4.12 \\
WC6 & -4.75 & -4.42 \\
WC7 & -4.80 & -4.18 \\
WC8 & -5.32 & -4.48 \\
WC9 & -5.57 & -5.13 \\
\hline
\end{tabular}

at least one star with a known distance modulus was available per subtype, this could be used to derive the apparent distances for all other WR stars of the same subtype, thus allowing to significantly enlarge the sample of studied stars. Of course the underlying assumption is rather arbitrary and already the existing scatter, especially seen in the Galactic and M31 WN stars (Hamann et al. 2006; Sander et al. 2014), clearly questioned this assumption. Now, with the distance moduli based on Gaia DR2, we can simply obtain the absolute magnitudes of all via

$M_{v, \text { new }}=M_{v, \text { old }}-\Delta$ D.M.,

and check how valid the assumption of a roughly constant $M_{v}$ per subtype is.

The so obtained distribution of $M_{v}$ vs. subtype is shown in Fig. 8 and Table 2, where we also denote the result from Sander et al. (2012) in gray for comparison. Only the WC4 stars essentially keep their mean $M_{v}$ while all other subtypes now have higher mean values. There are two basic reasons for this: First, for several subtypes there was just one star with a "known" distance in the 2012 sample. This yielded reliable results as long as this distance turns out to be confirmed by Gaia, such as for WR144, where the distance inferred from the parallax seems to verify the possible membership of Cyg OB2 claimed by Lundstrom \& Stenholm (1984) including their distance modulus. However, in other cases such as the WC7-star WR68, even the possible membership to the Cir OB1 association did not help, as the distance to the association has a significant uncertainty as different studies came to different results in the past. Thus the absolute magnitude inferred from the Gaia-based distance now increases by almost $1 \mathrm{mag}$, significantly affecting the calibration. 
Another issue now revealed with the help of the Gaia DR2 distances, is the internal spread in $M_{v}$, putting a general question mark behind this method. While for the WC4 subtype the results in Fig. 8 seem to hint at a more or less common $M_{v}$, other subtypes show a huge spread in intrinsic magnitudes. As is evident in Fig. 8, this spread is clearly present for all subtypes between WC5 and WC9, with an exception for the WC4 subtype, which could be possibly due to low number statistics. This would be in line with the picture that we see for example for the WC8 subtype: in Sander et al. (2012), WR135 was the only WC8 star with a "known" distance. While the $M_{v}$ with the distance based on Gaia DR2 only changed by $0.04 \mathrm{mag}$, three other WC8 stars are now available, all showing considerably larger values of $M_{v}$. In total, we can confirm the earlier suspicion that an $M_{v}$ calibration can at best provide rough estimates for the resulting luminosities when applied to an individual star and thus should be handled with great care.

\subsection{Notable objects}

In this section we comment on specific targets that are especially affected by the revision due to the new distance information:

WR 15 . With a luminosity of $\log L / L_{\odot}=5.99$, the WC6 star WR 15 is the most luminous star in our sample, not accounting for the strange and slightly suspicious transition type star WR 126 which is discussed below. In contrast to some of the other highly luminous WC stars in our sample, it has a rather low relative parallax error of only $9 \%$, leading to a robust distance of $3.0 \pm 0.25 \mathrm{kpc}$. WR 15 is considered to be a possible member of a potential concentration of OB stars termed Anon Vel b by Lundstrom \& Stenholm (1984), for which they also provide a distance of $2.5 \mathrm{kpc}$ following Bassino et al. (1982). The results based on Gaia DR2 are now more in line with the original suggestion by Muzzio (1979) that WR 15 could be associated with group of slightly more distant OB stars at about $3.2 \mathrm{kpc}$.

WR 59. WR 59 is a WC9d star and suspected binary candidate due to weak absorption features in the optical spectrum (Williams et al. 2005). WR 59 underwent the most drastic luminosity revision with now $\log L / L_{\odot}=5.76$, a shift by +0.86 dex. The reason of the previous underestimation is due to the $M_{v}$-calibration discussed in Sect. 3.4 that was applied as there was no information on the distance earlier. As this star turns out to have a very similar position in the HRD as the CWB WR 65 (see below), we mark it with a warning in the results Table 1.

WR 65. Another WC9d star is WR 65, which was suspected to by a binary due to optical absorption features by Williams et al. (2005) and later classified as CWB by Oskinova \& Hamann (2008) due to significant X-ray emission. In Sander et al. (2012), we excluded WR 65 from the $M_{v}$ calibration due to the high value of $M_{v} \approx-7$ one would obtain in the case of trusting the supposed association membership to Cir OB1. The distance based on Gaia DR2 now yields $M_{v}=-7.09$, thus indirectly confirming the membership, but also pointing toward a significant contribution of the companion. In this case, the underlying spectral analysis would not yield the correct stellar parameters, which is why we mark this star in Table 1 with a warning, similar to WR 59.

WR 88. WR 88 was classified as another WC9 stars until Williams et al. (2015) revealed WN features in the spectrum. A detailed modeling of the atmosphere by the same authors identifies WR 88 as a WC9/WN8 transition-type star with $X_{\mathrm{C}}=0.07$, a carbon abundance lower typical for WC stars, but already



Fig. 9. HRD with highlighted WO star positions: The yellow symbols indicate the positions using the stellar parameters from Sander et al. (2012) with the revised distances. The purple symbols use the same distances, but apply the stellar parameters from Tramper et al. (2015), including the result for the WO3 star WR 93b which was not covered in Sander et al. (2012).

Table 3. Revised stellar parameters of the single Galactic WO stars based on the atmosphere models used in Tramper et al. (2015) and the Gaia DR2 based distances from Bailer-Jones et al. (2018).

\begin{tabular}{lccc}
\hline \hline & WR 93b & WR 102 & WR 142 \\
\hline Subtype & WO3 & WO2 & WO2 \\
$T_{*}{ }^{a}(\mathrm{kK})$ & 160 & 210 & 200 \\
$v_{\infty}{ }^{a}\left(\mathrm{~km} \mathrm{~s}^{-1}\right)$ & 5000 & 5000 & 4900 \\
$X_{\mathrm{He}}{ }^{a}$ & 0.29 & 0.14 & 0.26 \\
$X_{\mathrm{C}}{ }^{a}$ & 0.53 & 0.62 & 0.54 \\
$X_{\mathrm{O}}{ }^{a}$ & 0.18 & 0.24 & 0.21 \\
$R_{*}\left(R_{\odot}\right)$ & 0.44 & 0.24 & 0.39 \\
$\log L / L_{\odot}{ }^{b}$ & $5.04_{-0.14}^{+0.17}$ & $4.98_{-0.06}^{+0.07}$ & $5.34_{-0.04}^{+0.04}$ \\
$\log \dot{M} /\left(M_{\odot} \mathrm{yr}^{-1}\right)^{b}$ & $-5.19_{-0.11}^{+0.13}$ & $-5.27_{-0.05}^{+0.05}$ & $-4.98_{-0.03}^{+0.03}$ \\
$M_{*}{ }^{b, c}\left(M_{\odot}\right)$ & $8.1_{-1.2}^{+1.9}$ & $7.3_{-0.5}^{+0.6}$ & $11.7_{-0.6}^{+0.7}$ \\
\hline
\end{tabular}

Notes. The parameters for the two WO2 stars based on the spectral analysis from Sander et al. (2012) can be found in Table 1. ${ }^{(a)}$ Taken from Tramper et al. (2015). ${ }^{(b)}$ Errors based on uncertainties of Gaia DR2 parallaxes converted into distances by Bailer-Jones et al. (2018). ${ }^{(c)}$ Masses are calculated from luminosity after Langer (1989) using their $M-L$ relation for WC stars.

depleted nitrogen $\left(X_{\mathrm{N}}=0.003\right)$ and slightly enriched oxygen $\left(X_{\mathrm{O}}=0.004\right)$. To preserve a homogenous sample, we keep the stellar parameters from Sander et al. (2012) in Table 1, but assign the revised spectral type. Unlike most transition-type stars, the WC9 features in WR 88 are clearly dominant here, so we also keep this object in the bucket of the WC9 stars for the discussions and plots, especially as $T_{*}$ stays the same in both studies. 
Rescaling the stellar parameters derived by Williams et al. (2015) to the new Gaia distance for WR 88 leads to a luminosity of $\log L / L_{\odot}=5.33$, slightly lower than the value of 5.49 we obtain using our 2012 model. Consequently, the mass-loss rate is slightly lower as well (-4.74 vs. -4.62$)$ and the inferred stellar mass drops from about $15 M_{\odot}$ to $11 M_{\odot}$. While these differences are not dramatic and thus do not affect our general conclusions, they underline our initial statement that for a detailed study of a particular object more tailored models should be used.

WR 93b. The WO3 star WR 93b was not analyzed by Sander et al. (2012) due to the lack of available spectra at that time. Using the atmosphere parameters from Tramper et al. (2015), we have calculated the revised radius, luminosity, mass-loss rate, and stellar mass based on the new distances from Bailer-Jones et al. (2018). The results for WR 93b and the other two single Galactic WO stars are listed in Table 3 with the HRD positions being shown in Fig. 9. As these values are from a different set of stellar atmosphere models and the results for the WO2 stars differ greatly between the two approaches as we discuss below, we list them separately and do not include WR 93b in the other diagrams which are solely based on the 2012 sample.

WR 102 and WR 142. The WO2 stars WR 102 and WR 142 shared a pretty similar position in the HRD in Sander et al. (2012). Now, with the updated distances, they mutually differ more in their respective luminosity, with WR 102 being shifted downwards by about 0.1 dex and WR 142 shifted upwards by more then 0.2 dex in luminosity, implying a current mass of about $29 M_{\odot}$, making the star a potential progenitor of a massive stellar black hole. Tramper et al. (2015) analyzed the two WO2 stars with the help of new spectra and derived more fine-tuned abundances, yielding quite similar stellar temperatures, but different luminosities of $\log L / L_{\odot}=5.45$ for WR 102 and 5.39 for WR 142. One would expect that this is mostly due to the different distances assumed for the objects at that time, but rescaling their results to the new distances based on Gaia DR2 would now lead to $\log L / L_{\odot}=4.98$ for WR 102 and 5.34 for WR 142. For both stars, this is about 0.6 dex lower than the revised results based on our models which we illustrate in Fig. 9.

Such a huge discrepancy is stunning and raises the question for their origin. Are the different stellar atmosphere models responsible, of are there other issues, for example in the proper reconstruction of the SED, which differs also in terms of technique between Sander et al. (2012) and Tramper et al. (2015). In Sander et al. (2012), we used additional photometry (Smith $b$ and $v$ in the optical, JHK in the near-IR) to constrain the SED, while Tramper et al. (2015) derived a Johnson $V$-band magnitude from their spectral observation that was then compared to the literature to obtain a flux calibration. We carefully revisited our spectral analyses for both stars and could not find any issues in our SED reconstruction. Unfortunately, the models in Tramper et al. (2015) do not account for Fe ions higher than $\mathrm{Fe} x$, which play a significant role in the temperature regime of these stars, but whether this can account for the huge discrepancy remains unclear.

The lower luminosities resulting from the updated distances applied to the models from Tramper et al. (2015) interestingly have only a moderate effect on the inferred mass-loss rates with about 0.2 dex difference for WR 142 and less than 0.1 dex difference for WR 102. On the other hand, the consequences for the stellar masses are dramatic as they sensitively depend on the luminosities. A comparison of the masses for WO2 stars between Tables 1 and 3 reveal that those inferred from our models are more than a factor of two higher. As we do not see an issue with our spectral analysis from Sander et al. (2012) apart from the fine-tuning of the abundances, we use the revised parameters from Table 1 for the discussions throughout the rest of this work.

WR 142 was the first WO star to be detected in X-rays with faint, but hard spectrum (Oskinova et al. 2009; Sokal et al. 2010). X-rays in single WC stars are not measured, but neither are there spectral hints for binarity, nor is the inferred $L_{\mathrm{X}} \approx 1.3 \times 10^{31} \mathrm{erg} \mathrm{s}^{-1}$ high enough to make WR 142 a candidate for a CWB. As WO winds are weaker compared to WC winds from stars with a similar luminosity (cf. Fig. 5), this might explain why X-rays can be measured for a putatively single star here while they cannot for the WC single stars with more dense winds.

WR 119. With $\log L / L_{\odot}=4.7$, the WC9d star WR 119 is now the least luminous star in our sample, being shifted down by 0.5 dex compared to our 2012 paper. There is considerable uncertainty of about $25 \%$ in the underlying parallax measurement, but even if the luminosity would be higher by about 0.3 dex, WR 119 would still be among the few WC stars marking the bottom end of the luminosity range. The star was part of the recent variability study by Desforges et al. (2017), but did not show any particularly special behavior compared to other WC9d stars.

WR 126. For WR 126, the luminosity was significantly revised from $\log L / L_{\odot}=5.43$ to $\log L / L_{\odot}=6.07$, making it the most luminous object in our sample. It is a somewhat odd outlier classified as WC5/WN, showing a spectral appearance with dominant WC features, but also prominent nitrogen lines, which is very rare as other WC stars do not show any trace of nitrogen while most transition-type stars have dominating $\mathrm{WN}$ features and are thus classified as WN/WC instead of WC/WN. While there is so far no clear evidence for binarity, the high luminosity combined with the relatively weak emission lines are suspicious. The WC/WN star might be part of a binary or multiple system, which would lead to a dilution of emission lines and an overestimation of the luminosity of the WR component. Given the large distance between 7.6 and $11.6 \mathrm{kpc}$ according to the Bayesian method from Bailer-Jones et al. (2018), about 2-3 times more than previously assumed, WR 126 might even be a small, unresolved cluster harboring a WC as well as a WN star instead of a transition-type object.

WR 145. WR 145 is a $\mathrm{WR}+\mathrm{O}$ binary system with a WN7/WCE transition-type primary. Using optical spectra, Muntean et al. (2009) derived a solution for the system with $M_{\mathrm{WR}}=18 M_{\odot}$ and $M_{\mathrm{O}}=31 M_{\odot}$. Applying the technique from Demers et al. (2002), they splitted the spectral components and determined the companion to be an0 $\mathrm{O} 7 \mathrm{~V}((\mathrm{f}))$. Moreover, the system is the brightest WR X-ray source in Cyg OB2 and was recently studied by Rauw et al. (2015), who deduced that the bulk of the X-ray emission might stem from the O-star companion while only a smaller amount originates in the wind-wind collision zone. A rough estimate based on the new Gaia distance gives $L_{\mathrm{X}} \leq 10^{33} \mathrm{erg} \mathrm{s}^{-1}$.

WR 150. The WC5 star WR 150 is the sample star with the smallest measured parallax of only $\varpi=0.024$ mas. Its error is $\sigma_{\varpi}=0.025$ mas, making it the only star in our sample which has a relative error that is as large as the measurement itself. A simple inversion of the parallax would place the star at a distance of more than $40 \mathrm{kpc}$, way outside the Milky Way. The Bayesian method by Bailer-Jones et al. (2018) gives a distance between 8.3 and $11.9 \mathrm{kpc}$, which seems to be more likely as it would place 


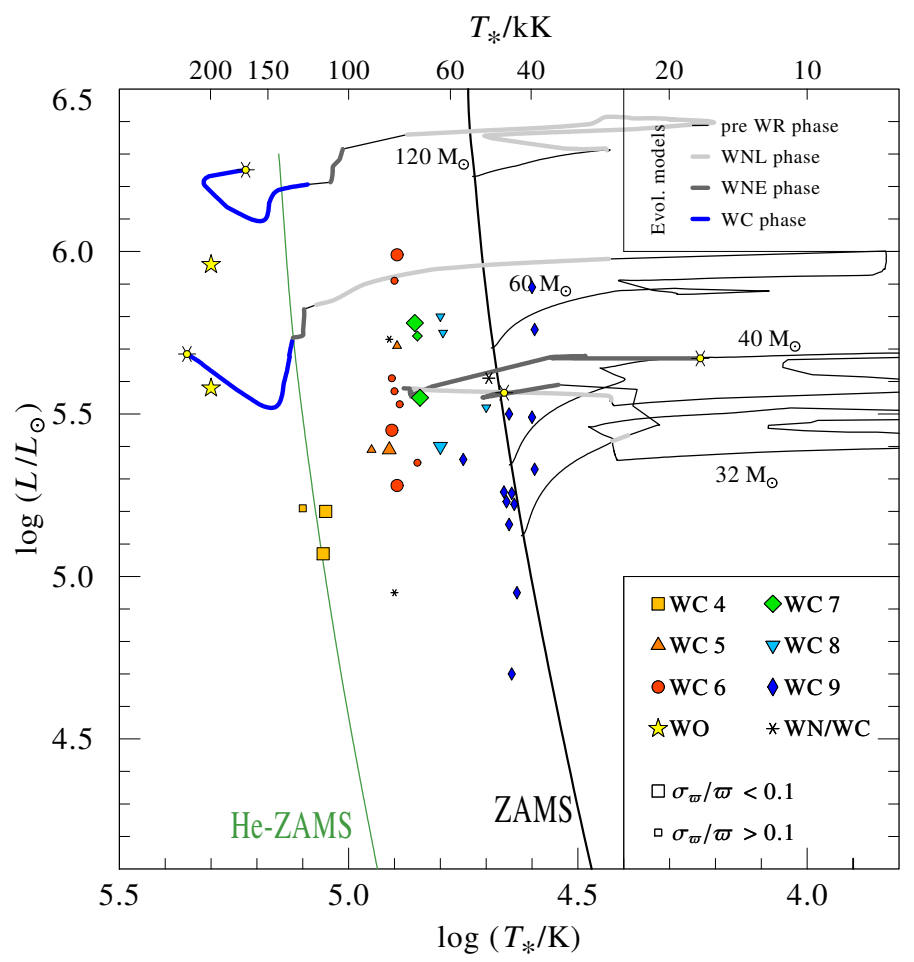

Fig. 10. HRD with the WC \& WO star positions compared to the evolutionary tracks from Ekström et al. (2012) without initial rotation. The thick lines indicate the WR phases of the tracks.

WR 150 more or less in an outer spiral arm (cf. Fig. 1) and leads to a reasonable luminosity of $\log L / L_{\odot}=5.86$.

\section{Discussion of the evolutionary status}

\subsection{Comparison with evolutionary tracks}

In order to form a classical, helium-burning Wolf-Rayet star, a star needs to lose its outer, hydrogen-rich layers. This can be done essentially in two ways: One possibility is that the star peels off its outer layers via intrinsic mass loss. The latter is believed to originate in either stellar winds ("Conti scenario", named after Conti 1976), or by episodic, massive outbursts due to instabilities (e.g. Langer et al. 1994), or continuum-driven eruptions (e.g. Smith \& Owocki 2006). Depending on the initial mass, both mechanisms might play a role during the lifetime of a massive star. Alternatively, a WR star can be produced in a binary system where Roche lobe overflow (RLOF) can be an important mass loss channel for the donor star (e.g. Paczyński 1967). However, so far, only a few WR stars have been convincingly suggested to have formed via binary interaction (e.g. Groh et al. 2008; Shenar et al. 2016).

In Figs. 10 and 11, we compare the revised positions of the Galactic WC stars in the HRD with the Geneva single-star evolution models for $Z=0.014$ from Ekström et al. (2012) without and with initial rotation, respectively. The evolutionary tracks are color-coded to highlight WR stages, following the usual assumption that these could be identified from surface compositions. A star with $T_{*}>25 \mathrm{kK}$ is considered a WR star if the hydrogen abundances is $X_{\mathrm{H}} \leq 0.4$. It is marked as a hydrogen-rich WN until $X_{\mathrm{H}} \leq 0.05$. Below this abundance, we mark the WR as a hydrogen-free $\mathrm{WN}$, or as $\mathrm{WC} / \mathrm{WO}$ if $X_{\mathrm{C}}>0.2$. While the precise limits for the abundances and temperature ranges differ, these "buckets" are essentially corresponding to what has been

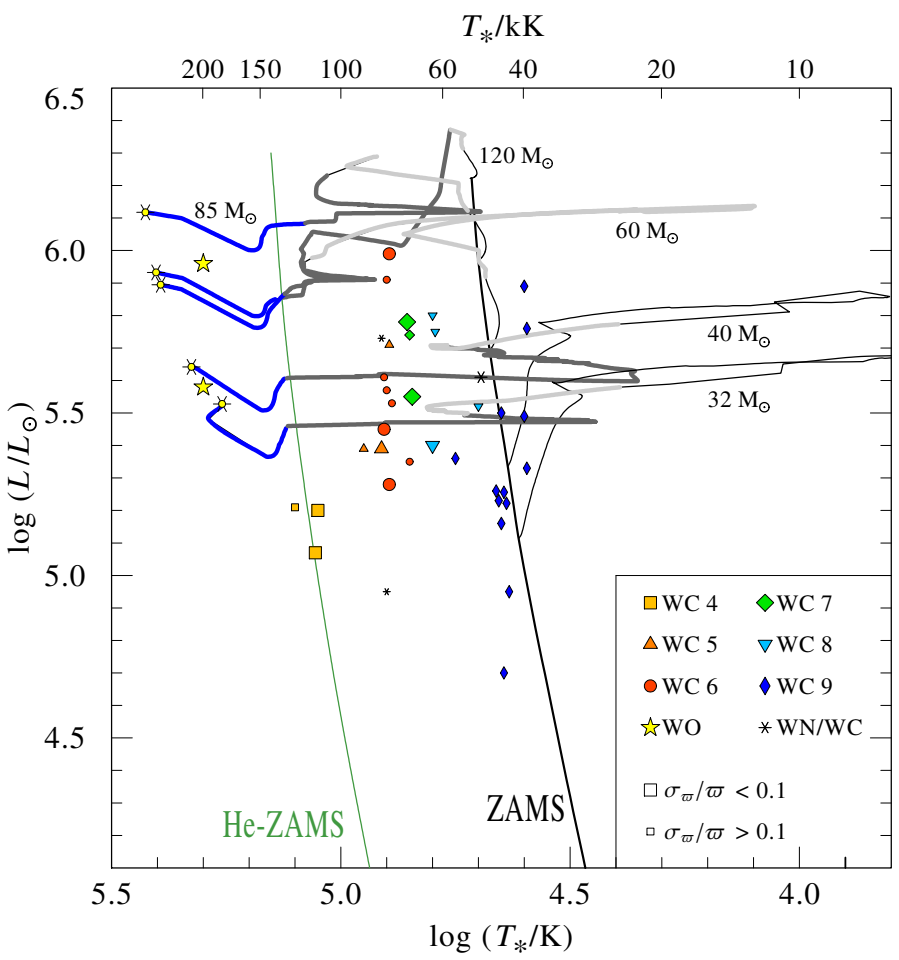

Fig. 11. HRD with the WC \& WO star positions compared to the evolutionary tracks from Ekström et al. (2012) with an initial rotational velocity of $0.4 v_{\text {crit }}$. The thick lines indicate the WR phases of the tracks.

labeled as WNL and WNE stars in an evolutionary sense (cf. e.g. Hamann et al. 2006; Sander et al. 2012; Georgy et al. 2012). However, since the terms "late" (WNL) and "early" (WNE) originally refer to the spectral type and the study of WR populations at lower metallicities have shown that the finding that early-type WN stars are hydrogen-free does not hold there (e.g. Hainich et al. 2014, 2015; Shenar et al. 2016), we refrain from over-stressing this potentially confusing terminology.

Assuming single-star evolution, the models with initial rotation are much better in reaching the area of the HRD covered by the WC stars than their counterparts without rotational mixing, which could explain only the objects with the highest luminosities. Nevertheless, also the models accounting for rotation do not reach $\log L / L_{\odot}<5.3$. While several stars have higher luminosities after the current revision, various ones remain in this "lower" luminosity regime, in particular all studied WC4 and some of the WC9 stars. Thus the problem already discussed by Georgy et al. (2012) for the original 2012 results remains, namely that the low luminosity WC stars would require higher mass-loss during their previous evolutionary stages. The Geneva models by Ekström et al. (2012) use the formula by Nugis \& Lamers (2000) for all WR stages except for the WNh stage (also termed WNL stage as these mostly co-incides with late WN subtypes for solar metallicity), where the recipe from Gräfener \& Hamann (2008) based on a set of hydrodynamically-consistent models is implemented. As denoted by Georgy et al. (2012), these theoreticallydriven approach leads to lower mass-loss rates in the WNh stage than usually inferred by empirical studies of WR stars (e.g. Hamann et al. 2006; Liermann et al. 2010).

Using the Bonn Evolution Code (BEC), Yoon (2017) argues that mass-loss rates slightly increased by a factor of $1.58 \mathrm{com}-$ pared to Nugis \& Lamers (2000) would be enough to explain WC and WO stars found with $\log L / L_{\odot}<5.3$. Such a raise in the mass-loss rate could be achieved by assuming a lower clumping 


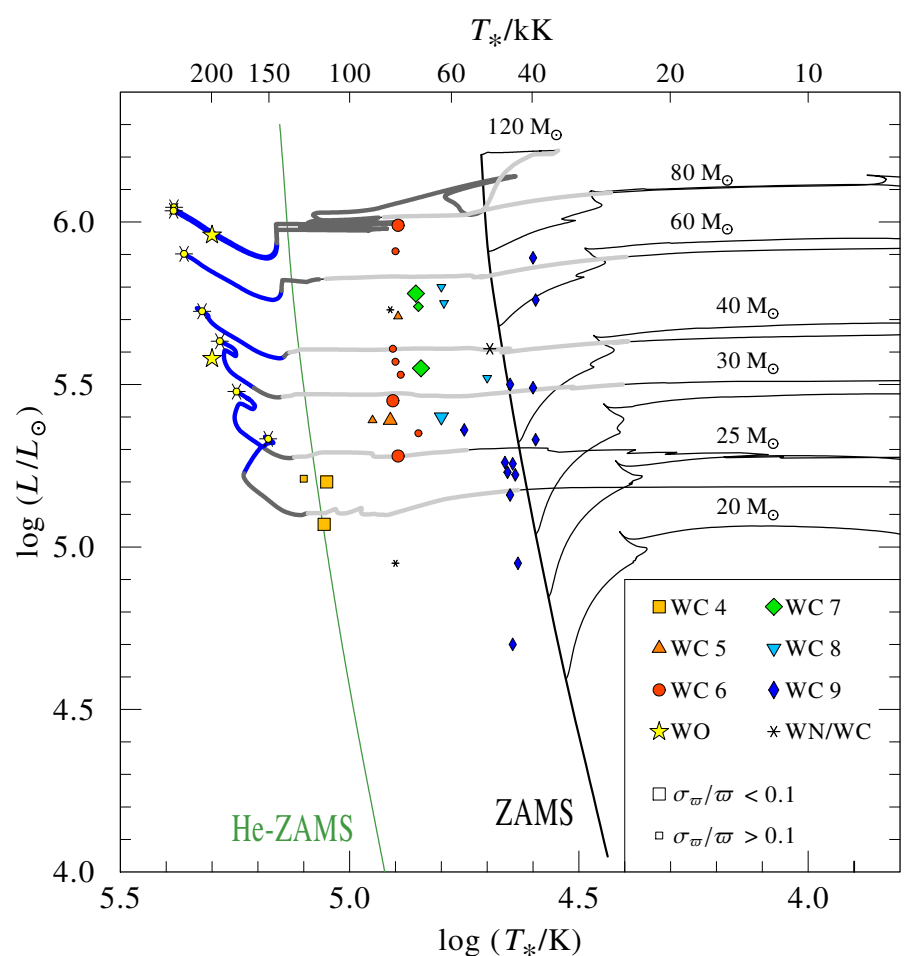

Fig. 12. HRD with the WC \& WO star positions compared to the evolutionary tracks from Chieffi \& Limongi (2013) with an initial equatorial rotational velocity of $v_{\text {rot,ini }}=300 \mathrm{~km} \mathrm{~s}^{-1}$. The thick lines indicate the WR phases of the tracks.

of $D=4$ instead of $D=10$. This would raise questions regarding a sufficient radiative wind driving. Atmosphere models where the hydrodynamic equation of motion is consistently included, in particular the WC results from Gräfener \& Hamann (2005), but also results for other types of hot stars (e.g. Gräfener \& Hamann 2008; Sander et al. 2017; Krtička \& Kubát 2017), point rather toward $D \geq 10$.

To study the how sensitive our conclusions are to the different choice of stellar evolution models, we compare our results in Fig. 12 to the tracks accouting for rotation from Chieffi \& Limongi (2013) obtained with the Frascati Raphson Newton Evolutionary Code (FRANEC). Their evolutionary models are calculated for the same initial metallicity of $Z=0.014$ as the Geneva models. Nevertheless, the FRANEC models differ in several details from the Geneva models, in particular the treatment of mixing including the assumptions for the initial rotation which is set to a fixed initial value of $v_{\text {rot,ini }}=300 \mathrm{~km} \mathrm{~s}^{-1}$ here compared to $0.4 v_{\text {crit }}$ in the Geneva models. While there are significant differences in terms of the shape of the tracks between Figs. 11 and 12, the most noticeable result of the FRANEC models is probably the lower initial mass of only $20 M_{\odot}$ required to reach the WR stage. However, one should keep in mind that none of the models up to $M_{\text {ini }}=30 M_{\odot}$ reaches our typical WC carbon mass fraction of $X_{\mathrm{C}}=0.4$ but instead end with $X_{\mathrm{C}} \approx 0.17$ (cf. Sect. 3.1). Thus, the WC phase of the tracks indicated in Fig. 12 is defined as $X_{\mathrm{C}}>0.15$ which should still provide a WC-type spectrum.

Despite the lower luminosities reached by the FRANEC models, the low carbon abundances of their lower mass tracks reveal that they essentially show the same discrepancies as the Geneva tracks. Not accounting for the temperature difference as this might be due to inflation which we discuss below, they especially cannot reproduce the evolved status of the WC stars with $\log L / L_{\odot}<5.4$ as their carbon surface mass fractions are higher.

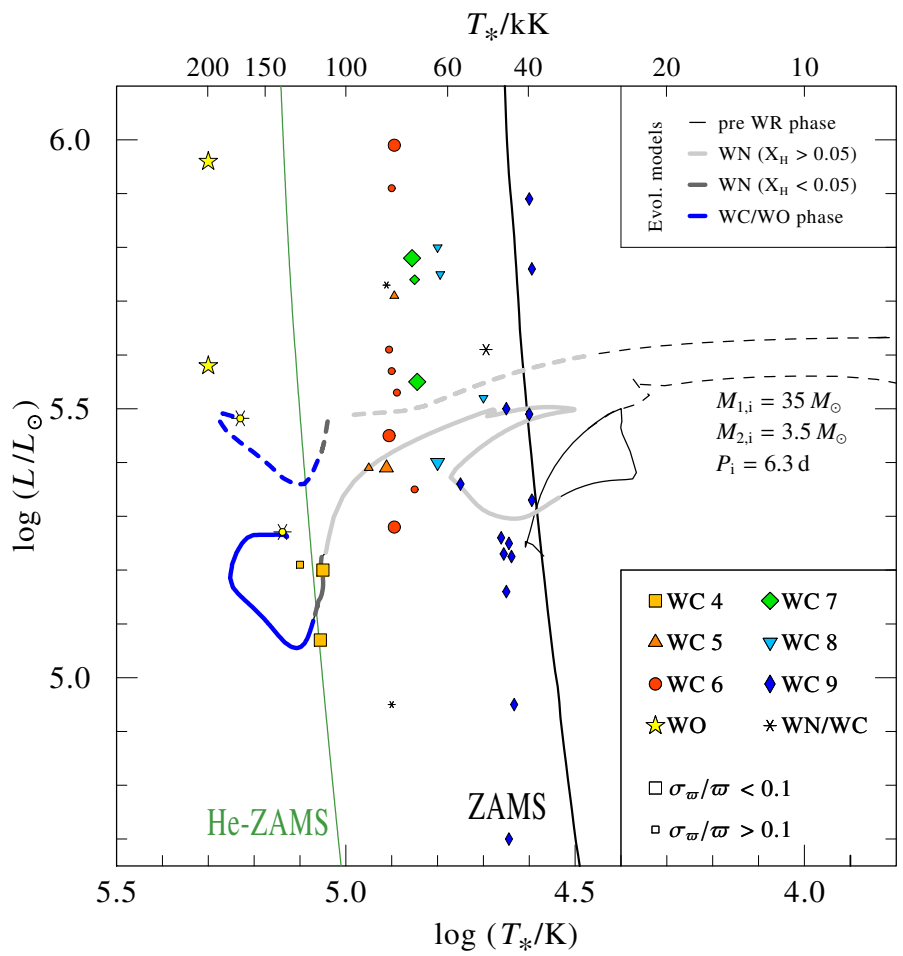

Fig. 13. HRD with the WC \& WO star positions compared to a BPASS binary evolution track. Only the evolution of the primary is shown, as the secondary has a way lower mass and remains on the main sequence at $\log L / L_{\odot} \approx 2.2$ during the whole lifetime of the primary. For comparison, also the corresponding single star evolutionary track for the primary is shown as a dashed line. The different colors indicate the different WR phases, defined via surface abundances.

A particular noticeable shortcome here are the WC4 stars with $X_{\mathrm{C}} \geq 0.4$. Thus the FRANEC models give some hints how the WR stage could be reached by initial masses as low as $20 M_{\odot}$, that is just above the observed upper limit of type II-L and II-P supernova ( $\mathrm{SN}$ ) progenitors. However, similar to the Geneva models they do not provide a viable scenario where enough mass is removed or processed such that the observed lower-luminosity WC stars is reproduced.

Other ways to remove the necessary amount of mass without invoking a binary scenario would be episodic mass-loss during a luminous blue variable (LBV) phase or higher mass-loss during the red supergiant (RSG) stage. Especially in the "lower" luminosity range, the stars might pass through the RSG stage, and calculations by Vanbeveren et al. $(1998,2007)$ have demonstrated that higher $\dot{M}$ could bring massive stars back to the blue side of the HRD. However, calculations by Meynet et al. (2015) revealed that higher mass-loss rates during the RSG stage essentially just lower the time of a star spent as a red supergiant, but do not increase the total amount of mass lost during this stage considerably. Thus, a higher $\dot{M}$ during the RSG stage would have little impact on the obtained (synthetic) WR population. LBVs could help to remove the necessary amount of mass as they appear at a relative wide range of luminosities (e.g. Humphreys et al. 2016; Smith et al. 2018). However, there is an ongoing debate whether LBVs are isolated as claimed by Smith \& Tombleson (2015) or associated with O-stars and RSGs as Humphreys et al. (2016) deduced based on a sample of LBVs in M 31 and M 33. Smith et al. (2018) have pointed out that, based on the new Gaia DR2 data, well known Galactic LBVs and LBV candidates with a WR-type spectrum at quiescence such as WR 31a (Hen 3-519) and WR 31b (AG Car) turn out to be at large distances and could thus not be associated 
with the O-stars projected near to them on the sky in the Car OB association.

As mentioned in the beginning of this section, binary evolution is an alternative channel to remove enough mass to reach the lower luminosities from our sample, as demonstrated for example by the low masses for type Ibc SN progenitors reached in the calculations by Yoon et al. (2010). While a detailed study for particular objects is beyond the scope of this paper, we illustrate in Fig. 13 that the positions of the WC4 stars can be reached with a binary evolution scenario. For this example we employ evolutionary tracks calculated with version 2.0 of the Binary Population and Spectral Synthesis (BPASS, e.g. Eldridge \& Stanway 2009; Eldridge et al. 2017) code. In addition to the physics involved in single-star evolution, BPASS also accounts for binary mass transfer. The models do not account for rotationally induced mixing or tidal interactions, but this is not necessary for this purpose. In our example, a binary system with a primary of $35 M_{\odot}$ and a secondary of $3.5 M_{\odot}$ (i.e., a mass-ratio of $q=0.1$ ) is shown, where the primary transfers mass onto the secondary when expanding after the end of its main sequence (MS) phase. Eventually this leads to the effect that the WR stage is reached at lower luminosities compared single star evolution, which is also depicted in Fig. 13.

Despite the success for the WC4 stars, the problem for the later subtypes, in particular the WC9 stars, remains even when invoking binary evolution. While scenarios can be found that cross this area of the HRD as WR stars, none of them has already reached the WC stage. As discussed in Sect. 3.2, the effective temperatures of stellar evolution models are based on a simplified treatment of the stellar atmosphere (cf. Groh et al. 2014) and thus the temperature discrepancy could be seen as kind of an "outer boundary problem" of the stellar evolution models as the later WC subtypes might have inflated envelopes. In particular, according to the results of Gräfener et al. (2012a), the winds of late WC stars with lower luminosity would need to be clumped with $D \geq 10$ for reaching the lower temperatures due to inflation, which is on the order of what is derived from electron scattering wings. Partially based on the WC results from Sander et al. (2012) and applying an approximate method to estimate the optical depth of the sonic point (cf. Vink \& Gräfener 2012), Gräfener \& Vink (2013) calculated a set of optically thick wind models and discovered that the WC and WO stars obey a relation of $P_{\text {rad }} / P_{\text {gas }} \approx 80$ at the sonic point. If a star is near the Eddington limit, a smooth connection between the outer stellar envelope and their wind models could only be achieved in two cases: In the first one, the sonic point is located below at hot $\mathrm{Fe}$ opacity peak, which would lead to a compact solution like we get for the WO and early WC subtypes. In the second one, there is an extended atmosphere, where the subphotospheric layers at the location of the sonic point, which is then at lower temperatures above the hot Fe opacity peak, are clumped. Gräfener \& Vink (2013) further argue that due to the concordance of their results with Glatzel (2008), strange-mode instabilities in the inflated layers might be the origin of WR wind clumping as such. The idea of extended or inflated envelopes for later WC subtypes is further backed by calculations for thick WR winds from Gräfener et al. (2017) who found no smooth wind solutions in this luminosity regime. In addition, McClelland \& Eldridge (2016) pointed out, on the basis of pure helium star evolution, that to reach the parameters of the WC9 stars with evolutionary models, inflation alone would not be sufficient as their model counterparts would still have nitrogen which is not observed. They suggest that additional mixing, for example by rotation, could help to process the nitrogen into different elements, thus removing it from the surface.
In total, we can conclude that while the status of the WC stars as evolved, core-helium burning object is beyond doubts, the details of the evolutionary path toward this stage still remain unclear to a significant degree. While a certain fraction even of the nowadays single WC stars might have a binary history, and some of them might have a yet undetected, low-luminosity companion, the evolutionary models from different groups can in principle provide single-star scenarios for WC stars with $\log L / L_{\odot} \gtrsim 5.4$, thus already covering about half of the studied sample. They do not reproduce the temperatures of the late WC subtypes, but this is also an issue for binary evolution scenarios. Adjustments of the mass-loss rates in certain evolutionary stages might improve the coverage of the HRD regions. Thus, we conclude that singlestar evolution is likely not the only, but still a viable channel to obtain WC and WO stars at Galactic metallicity.

\subsection{Evolutionary scenarios for different mass regimes}

Breaking single-star evolution down into different mass regimes, we have to note that there seems to be still an upper luminosity limit for WC stars, but now at $\log L / L_{\odot} \approx 6.0$ instead of the previously 5.6 deduced in Sander et al. (2012). Thus, for the most massive stars with an initial mass $M_{i}$ of more than $60-80 M_{\odot}$, the scenario inferred by Smith \& Conti (2008) and discussed in our 2012 paper is the prime candidate. Introducing the WNH notation (with captial $\mathrm{H}$ for distinction) from Smith \& Conti (2008) for luminous, hydrogen-rich $\mathrm{WN}$ stars, which are seen as corehydrogen burning, pre-LBV stars, the evolutionary sequence in the uppermost mass regime reads

$\mathrm{O} \rightarrow \mathrm{Of} / \mathrm{WNH} \leftrightarrow \mathrm{LBV}\left[\rightarrow \mathrm{WNL}_{\mathrm{H}-\text { reduced }}\right] \rightarrow$ SNIIn.

The bracket term suggests that a late-type WN stage with reduced, but not necessarily zero hydrogen could follow the LBV stage before the eventual SN explosion. Such stars with low hydrogen mass fractions are found among the WNL stars and with parameters close to the so-called "S Dor instability strip" (see, e.g. Sander et al. 2014) where various known LBVs are located during quiescence. Further evidence for such a scenario are the recent analysis of the progenitor spectrum of SN2015bh (Boian \& Groh 2018) revealing typical parameters of an LBV candidate and also the observation of SN2014C which turned from a type Ib into a type IIn SN during approximately one year, revealing the presence of a massive shell of hydrogen-rich material likely from an earlier, but still relatively recent outburst (Margutti et al. 2017).

For lower masses where $M_{i}$ is still above the upper mass limit for RSGs, the revised luminosities now lead to the conclusion that the WC stage is probably not skipped as originally speculated in Sander et al. (2012), thus leading to a sequence like

$\mathrm{O} \rightarrow \mathrm{Of} / \mathrm{WNH} \leftrightarrow \mathrm{LBV} \rightarrow \mathrm{WN} \rightarrow \mathrm{WC} \rightarrow \mathrm{WO} \rightarrow \mathrm{CC}$

with CC denoting core collapse. This collapse might come with a $\mathrm{SN}$ explosion, but there is also increasing evidence from both theoretical and observational side during the last years (e.g. Smartt 2015; Sukhbold et al. 2016; Adams et al. 2017) that a good fraction of massive stars might fail to explode as a SN but collapse quietly into a compact object, which in our mass regime would most likely be a black hole. In case of an exploding WO star, the corresponding supernova would have to be of type Ib or Ic, depending on the amount of helium left at the time of collapse. Nevertheless, SN explosion models that try to reproduce the observed properties of such a SN with a Wolf-Rayet progenitor turn out to be difficult and highly asymmetric explosion scenarios need to be invoked (e.g. Dessart et al. 2017). 
Table 4. Absolute Johnson magnitudes for selected WC and WO stars scaled to the same luminosity of $\log L / L_{\odot}=5.5$.

\begin{tabular}{lcccc}
\hline \hline & WR 102 & WR 52 & WR 13 & WR 103 \\
\hline Subtype & WO2 & WC4 & WC6 & WC9 \\
$M_{U}$ & -3.0 & -5.4 & -5.7 & -7.2 \\
$M_{B}$ & -1.8 & -4.2 & -4.5 & -6.2 \\
$M_{V}$ & -1.7 & -4.0 & -4.3 & -6.1 \\
\hline
\end{tabular}

Notes. Corresponding magnitudes for different luminosities can be obtained by adding or subtracting one magnitude per 0.4 dex shift in luminosity.

The idea, that the bulk of massive stars with $M_{i} \gtrsim 18 M_{\odot}$ might collapse silently, or at least without a bright SN outburst, is observationally supported from two sides: first, apart from debates about type IIn $\mathrm{SNe}$, no type II SN progenitor with $\log L / L_{\odot}>5.1$ has been found (Smartt 2015). Secondly, there is the lack of known progenitors for type Ibc SNe with only one possible progenitor candidate discovered very recently (Van Dyk et al. 2018). This has lead to the idea that the bulk if not all type Ibc SNe could stem from progenitors with $M_{i}=$ 8-20 $M_{\odot}$ in a binary system (Bersten et al. 2014; Eldridge et al. 2015; Smartt 2015). However, an upper mass cut for SNe at about $18 M_{\odot}$ would be challenging with regards to our current picture of nucleosynthesis (Brown \& Woosley 2013), enforcing fine-tuning on some nuclear reactions and also constraints on wind mass-loss rates to avoid a nonobserved overabundance of carbon.

The nondetection of type Ibc SNe progenitors has lead to magnitude limits that have been compared to potential progenitors. For the type Ic SN 2004gt, Gal-Yam et al. (2005) derived $M_{B}>-6.5$ and $M_{V}>-5.5$. Recently, Johnson et al. (2017) derived much stricter limits of $M_{U}>-3.8, M_{B}>-3.1$, and $M_{V}>-3.8$ for the SN 2012fh, which was also classified as type Ic but with some uncertainty due to its rather late discovery. In Table 4, we have compiled the absolute broadband magnitudes for three WC and WO stars which have rather typical parameters with regard to their spectral subtype. To allow an easy comparison, their magnitudes have been scaled such as if all stars would have the same $\log L / L_{\odot}=5.5$. Assuming this luminosity, a WC6 progenitor would fit within the derived limits for SN 2004gt, while a similar progenitor for SN 2012fh would need to be as faint as $\log L / L_{\odot} \lesssim 4.7$. However, the first column of Table 4 shows that absolute magnitudes decrease significantly when considering WO stars. Just considering the photometry, both SN 2004gt and SN 2012fh could easily have a WO progenitor, but not any kind of WC progenitor unless their luminosity was really low. The complex detectability of SNe Ibc progenitors and the result that progenitors with higher masses must not be optically brighter than those lower masses was already noted by Yoon et al. (2012) when comparing evolutionary tracks with the WC results from Sander et al. (2012).

Given the fact that especially type Ic supernova progenitors need to be depleted not only in hydrogen, but also in helium, WO stars are the only proper candidates with regard to chemical composition. This is also supported by theoretical studies from Groh et al. (2013), who calculated synthetic spectra for a set of stellar evolution models and deduced that - assuming there is no silent collapse - all type Ic SNe in their calculations stem from WO stars while most type Ib SNe stem from late-type WN stars with only a smaller WO contribution. While WN stars will be discussed in a subsequent paper, we can already conclude that the current observational constraints of type Ibc SNe and the theoretical considerations point to a picture where WC stars are not SN progenitors, but only WO stars are. This would be qualitatively in line with the calculations from Groh et al. (2013), but it remains to be seen whether the remaining amount of approximately $X_{\mathrm{He}}=0.25$ could be "hidden" in type Ic $\mathrm{SNe}$, which is a matter of debate (e.g. Hachinger et al. 2012; Dessart et al. 2012; Piro \& Morozova 2014; Prentice et al. 2018). Frey et al. (2013) demonstrated that enhanced convection could severely deplete helium, reducing it down to $X_{\mathrm{He}} \approx 0.15$ for their $27 M_{\odot}$ helium star model, not even accounting for WR mass-loss. With $X_{\mathrm{He}}=0.15$ derived by Tramper et al. (2015), the WO2 star WR 102 seems to be the most promising progenitor candidate for a type Ic SN so far.

Backed up by the identification of RSGs with $M_{i} \lesssim 18 M_{\odot}$ as type II supernova progenitors (e.g. Smartt 2009, 2015), the lowluminosity part of the WC sample is hard to explain via classical single-star evolution. In theory there is the concept of chemically homogeneous evolution (e.g. Maeder 1987; Langer 1992; Heger \& Langer 2000), but this should be less efficient at higher metallicities due to the stronger stellar winds leading to mass and angular momentum loss, eventually spinning down the stars and thus reducing the mixing (e.g. Yoon \& Langer 2005; Brott et al. 2011; Cui et al. 2018).

For the remaining regime between $M_{i} \gtrsim 18 M_{\odot}$ and the upper mass limit of RSGs, a post-RSG scenario would be the classical choice, but potentially enhanced by a low-luminosity LBV stage during the path of the star back to the blue part of the HRD, that is

$\mathrm{O} \rightarrow \mathrm{RSG} \rightarrow \mathrm{WNL}[\leftrightarrow \mathrm{LBV}] \rightarrow \mathrm{WNE} \rightarrow \mathrm{WC} \rightarrow \mathrm{WO} \rightarrow \mathrm{CC}$

The lower luminosities for the bona fide LBVs and LBV candidates obtained with the distances inferred from Gaia DR2 parallaxes by Smith et al. (2018) would support such an additional LBV stage, but their isolation argued by the same authors would immediately contradict this scenario. Moreover, the luminosities derived by Smith et al. (2018) are based on variety of different original luminosity determinations. As we see, e.g., from the conflicting results for the WO2 stars (cf. Sect. 3.5), a robust luminosity determination requires not only an accurate distance determination, but also a sufficient current-generation stellar atmosphere analysis. Such research has so far only been performed for a small subsample of LBVs and LBV candidates.

Hydrogen-free WR stars are also the progenitor candidates for long-duration Gamma Ray Bursts (L-GRBs), which are interpreted as so-called "failed SNe" (Woosley 1993; MacFadyen \& Woosley 1999), in contrast to shorter GRBs which are attributed to white dwarf and compact object mergers as recently confirmed by the simultaneous detection of a gravitational wave and a GRB event (Abbott et al. 2017). The term "failed" might be misleading as it does not necessarily mean that there is no $\mathrm{SN}$, but that there is not enough neutrino energy for driving the explosion. However, as Bodenheimer \& Woosley (1983) argue, an explosion driven by other mechanisms (e.g., rotation, nuclear burning) can still occur at a slightly later stage. In fact there is strong observational evidence (e.g., Hjorth et al. 2003; Woosley \& Bloom 2006) for connections between type Ic SNe with broad lines - also termed "hypernovae" due to their high inferred kinetic energies - and L-GRBs.

For appearing as a type Ic $\mathrm{SN}$, the progenitor star must not have any hydrogen and also a significant helium depletion, bringing WC and especially WO stars into the focus. The present 
nondetection of SN Ibc progenitors in all cases with available pre-explosion images makes it unlikely that WR stars are the only progenitors (see, e.g., the discussion in Sect.5.1 in Smartt 2009). Less luminous and thus less massive "stripped" progenitors which may not appear as WR stars might be needed as well. Nonetheless, WC and WO stars should still feed this SN channel, if they explode at all. Moreover, apart from classical subdwarfs, the massive WR stars are the only well-studied objects in the hydrogen-free or nowadays more often termed "stripped-envelope" regime, while the inferred parameters for a lower mass regime (e.g., Vink 2017; Götberg et al. 2018) are highly uncertain, due to the lack of constraints for various ingredients in the underlying models, and can so far only be benchmarked by one object assumed to belong to these kind of stars, namely the so-called qWR ("quasi Wolf-Rayet") star HD 45166 (Groh et al. 2008). With sufficient mass loss, such stripped stars ranging from $M_{i} \lesssim 18 M_{\odot}$ would appear as WN stars and might evolve further to the WC stage. In our WC and WO sample, there is no clear evidence that any of the sample stars is the product of such a process, but especially for the WC9 objects with the lowest luminosities - in particular WR 119 - this could be an evolutionary channel that would have to explored in more detail.

Essentially for the same reasons as in the discussion about quasi-homogenous evolution, GRBs are found and expected to form at low metallicity (e.g., Yoon \& Langer 2005; Woosley \& Heger 2006; Yoon et al. 2006), explaining why they are often found at noticeable redshift. Nonetheless, GRB candidates in the form of fast-rotation WR stars have also been proposed and searched for at solar and near-solar metallicity (Petrovic et al. 2005; Vink et al. 2011; Gräfener et al. 2012b). In Sander et al. (2012), we discussed the two WO2 stars as potential GRB candidates due to the high rotational velocity adopted for reproducing their observed spectra. Later, Tramper et al. (2015) demonstrated that such a significant rotational velocity $\left(\sim 1000 \mathrm{~km} \mathrm{~s}^{-1}\right)$ is not needed to reproduce the observed broad line profiles for the Galactic WO2 stars. However, Shenar et al. (2014) showed that unless the wind significantly co-rotates, the emission line spectrum of a WR star with a dense wind is hardly affected, even if the star itself rotates with velocities up to $5000 \mathrm{~km} \mathrm{~s}^{-1}$. Although unlikely as it would require to invent a mechanism that has lead to spun-up of the star, this means that Galactic WR stars essentially would be able to "hide" their rotation to a normal spectral analysis, and methods like polarization measurements are required. As long as this has not been done, the possibility of an L-GRB during CC for Galactic WO stars like WR 102 and WR 142 might not be likely, but cannot be ruled out either.

\section{Summary and conclusions}

Building on the parallaxes from Gaia DR2 and the distances inferred by Bailer-Jones et al. (2018), we have revised the stellar and wind parameters of the single Galactic WC and WO stars. Focussing on the impact of the new distances, only parameters depending on the distances were updated compared to the analysis of Sander et al. (2012). The new results revealed that the assumption of roughly same $M_{v}$ per WC subtype is not justified, perhaps with the exception of the WC4 subtype. The luminosities of the Galactic WC stars are found to span a range from approximately $\log L / L_{\odot}=4.9-6.0$ with one outlier (WR 119) having $\log L / L_{\odot}=4.7$. The most luminous star in our sample is the WC/WN transition-type star WR 126 with $\log L / L_{\odot} \approx 6.1$, which might not be a single object.
Table 5. Mean WC/WO star parameters per subtype.

\begin{tabular}{crccccc}
\hline \hline Subtype & $\begin{array}{r}T_{*} \\
(\mathrm{kK})\end{array}$ & $\begin{array}{c}v_{\infty} \\
\left(\mathrm{km} \mathrm{s}^{-1}\right)\end{array}$ & $\begin{array}{c}R_{*} \\
\left(R_{\odot}\right)\end{array}$ & $\begin{array}{c}\log \dot{M}^{a} \\
\left(M_{\odot} \mathrm{yr}^{-1}\right)\end{array}$ & $\begin{array}{c}\log L \\
\left(L_{\odot}\right)\end{array}$ & $\begin{array}{c}M_{\mathrm{WC}}^{b} \\
\left(M_{\odot}\right)\end{array}$ \\
\hline WO2 & 200 & 5000 & 0.7 & -4.96 & 5.8 & 22 \\
WC4 & 117 & 3310 & 0.9 & -4.67 & 5.2 & 10 \\
WC5 & 83 & 2780 & 3.2 & -4.39 & 5.6 & 18 \\
WC6 & 78 & 2270 & 3.6 & -4.47 & 5.7 & 18 \\
WC7 & 71 & 2010 & 4.0 & -4.57 & 5.6 & 17 \\
WC8 & 60 & 1810 & 6.3 & -4.53 & 5.6 & 18 \\
WC9 & 44 & 1390 & 8.7 & -4.66 & 5.4 & 13 \\
\hline
\end{tabular}

Notes. ${ }^{(a)}$ All mass-loss rates are based on models from Sander et al. (2012) which are calculated with a clumping factor of $D=10{ }^{(b)}$ The masses of the WC stars are calculated from luminosities using the relation by Langer (1989).

Table 6. Suggested single-star evolution scenarios based revised WC/WO parameters.

\begin{tabular}{ll}
\hline \hline$M_{\mathrm{i}}\left(M_{\odot}\right)$ & Evolutionary path \\
\hline $8-18$ & $\mathrm{OB} \rightarrow \mathrm{RSG}[\rightarrow \mathrm{BSG}] \rightarrow \mathrm{SN}$ II \\
$18-35$ & $\mathrm{O} \rightarrow \mathrm{RSG} \rightarrow \mathrm{WNL}[\leftrightarrow \mathrm{LBV}] \rightarrow \mathrm{WN} \rightarrow \mathrm{WC}$ \\
& $\rightarrow \mathrm{WO} \rightarrow \mathrm{CC}$ \\
$35-80$ & $\mathrm{O} \rightarrow \mathrm{Of} / \mathrm{WNH} \leftrightarrow \mathrm{LBV} \rightarrow \mathrm{WN} \rightarrow \mathrm{WC} \rightarrow \mathrm{WO} \rightarrow$ \\
& $\mathrm{CC}$ \\
$>80$ & $\mathrm{O} \rightarrow \mathrm{Of} / \mathrm{WNH} \leftrightarrow \mathrm{LBV}[\rightarrow \mathrm{WNH}] \rightarrow \mathrm{SN} \mathrm{IIn}$ \\
\hline
\end{tabular}

Notes. The mass ranges should be taken as rough estimates inferred from comparing empirical luminosities with current evolutionary calculations instead of hard limits.

For comparison, we also performed the same calculations for the three Galactic WO stars analyzed by Tramper et al. (2015). The parameters of those WO2 stars, which could be obtained from both sources, turned out to differ significantly in luminosity and all parameters depending on $L$. Since both studies used rather different atmosphere models, only a complete revision of the spectral analysis with new atmosphere models that resolve the issues of the previously applied ones can help to resolve this discrepancy.

As a consequence of the new luminosities, also the massloss rates of the Galactic WC stars had to be revised. Adopting a (micro-)clumping factor of $D=10$ for the whole sample, they cover the range between $\log \dot{M}=-5.1$ and -4.1 , with a linear regression yielding $\dot{M} \propto L^{0.68}$. The two WO2 stars have mass-loss rates that are about 0.5 dex or 0.3 dex lower compared to WC stars with the same luminosity, depending on whether one uses the analyses from Sander et al. (2012) or Tramper et al. (2015). We also found an essentially linear relation between the modified wind momentum $D_{\text {mom }}$ and the revised WC luminosities, that is $D_{\text {mom }} \propto L$. Interpreting this with the mCAK theory would yield $\alpha_{\text {eff }}=1$, while Gräfener \& Hamann (2005) numerically obtained $\alpha_{\text {eff }} \approx 0$. We take this as a further confirmation that current CAK-like concepts essentially fail do describe WolfRayet winds. Obviously, a further investigation about the driving of WR winds is needed.

Mean WC parameters per subtype are listed in Table 5. We see that earlier WC subtypes haves higher $T_{*}$ and $v_{\infty}$. A general trend of increasing radii with later subtypes can be noticed. Regarding luminosities and masses, only the WC4 stars show considerably lower values while there is no clear trend between the later WC subtypes with the WC9 slightly falling out. This 
is due to the result that only a smaller part of WC9 stars has a higher luminosity, while the majority has $\log L / L_{\odot}<5.3$.

In this work we did not update any of the underlying atmosphere analyzes performed for the sample stars. Thus, for an indepth look at a particular object, we recommend to use the latest generation of atmospheres models, which have undergone various improvements since Sander et al. (2012). With regards to PoWR, an updated grids of atmosphere models with ready-touse synthetic spectra for WC stars at Galactic and LMC (not available in 2012) metallicity have been published on the PoWR website ${ }^{2}$.

Moreover, the situation in terms of radius and temperature discrepancies for earlier WC subtypes might be improved when a more accurate velocity and thus density stratification is assumed, which would require further updates of the atmosphere model code. Recently, Grassitelli et al. (2018) showed that it is possible to connect a stellar structure model smoothly to the hydrodynamically consistent model for a WC5 star from Gräfener \& Hamann (2005). While the calculation of such complex and time-consuming models is not an option when analyzing a whole population, a subset of representative models could provide valuable constraints such as velocity fields tailored to different WC subtypes.

The current evolutionary status of the Galactic WC and WO stars as evolved, core-helium burning objects is solid, but the path toward this status remains uncertain. Our revised luminosities set new constraints, and, together with our recent results, slightly change the picture compared to Sander et al. (2012). For $\log L / L_{\odot}>5.4$, current single star evolutionary calculations predict the formation of $\mathrm{WC}$ and $\mathrm{WO}$ stars at $Z_{\odot}$, although failing to reproduce the temperatures for the later subtypes, which might be due to the occurrence of inflated envelopes (e.g., Gräfener et al. 2012a, 2017). We conclude that while some of the stars in our sample might not have formed via the single star channel, the loss of mass without a binary companion is a viable channel to form $\mathrm{WC}$ and $\mathrm{WO}$ stars at $Z_{\odot}$. Suggested scenarios are summarized in Table 6 and discussed in more detail in Sect. 4. Binary evolution is required to explain the lower luminosity WC stars and could also be responsible for a fraction of the higher luminosity stars. The revision of wind mass-loss rates and better knowledge about episodic mass loss might still change this picture in the future, and could perhaps reopen the single star channel in the lower luminosity regime.

A particular challenging object is the WC9d star WR 119. With a luminosity of only $\log L / L_{\odot}=4.7$ and a current mass of approximately $6 M_{\odot}$, the star is likely a product of binary evolution, although there is no clear evidence for a close binary system. The system might be an interesting test case as it could be the evolved status of a so-called "stripped envolope" star, resulting from a primary with $M_{i}<18 M_{\odot}$ that lost its hydrogen envelope to a companion and eventually from a WN to a WC stage.

With masses between approximately 8 and $30 M_{\odot}$ for the remaining sample, the Galactic WC and WO stars are progenitors of massive black holes. The observed upper limit of $\log L / L_{\odot} \approx 5.1$ for type II SNe progenitors and only one possible detection of a type Ibc progenitor opens the possibility that most massive stars, including our sample stars, collapse silently at the end of their lifetime, that is without a visible SN outburst. However, if all WC stars evolve into a WO stage eventually, their absolute magnitudes in the $U, B$, and $V$ filters would be low enough to have escaped current progenitor detections. Thus, we conclude that lack of type Ibc SN progenitors does not necessar-

\footnotetext{
2 wWw.astro.physik.uni-potsdam.de/PoWR
}

ily mean that WR stars do collapse silently, although this scenario is evenly possible. Furthermore, the only class of classical Wolf-Rayet stars that would be viable progenitor candidates for type Ic SNe, are the WO stars.

Acknowledgements. The authors would like to thank the referee, C. Georgy, for fruitful comments and suggestions that helped to increase the quality of this paper. A.A.C.S. would further like to acknowledge helpful discussions and suggestions with P.M. Williams and J.S. Vink. This research has made use of the SIMBAD database and the VizieR catalog access tool, both operated at the CDS, Strasbourg, France. This work has made use of data from the European Space Agency (ESA) mission Gaia (https://www . cosmos.esa.int/gaia), processed by the Gaia Data Processing and Analysis Consortium (DPAC, https://www. cosmos.esa.int/web/gaia/dpac/ consortium). Funding for the DPAC has been provided by national institutions, in particular the institutions participating in the Gaia Multilateral Agreement. The first author of this work (A.A.C.S.) is supported by the Deutsche Forschungsgemeinschaft (DFG) under grant HA 1455/26 and would like to thank STFC for funding under grant number ST/R000565/1. T.S. acknowledges funding from the European Research Council (ERC) under the European Union's DLV_772225_MULTIPLES Horizon 2020 research and innovation program. V.R. is grateful for financial support from the Deutsche Akademische Austauschdienst (DAAD) as part of the Graduate School Scholarship Program. L.M.O. acknowledges support from the DLR under grant 50 OR 1809.

\section{References}

Abbott, J. B. 2004, PhD Thesis, University of London, University College London, UK

Abbott, J. B., Crowther, P. A., Drissen, L., Dessart, L., \& Martin, P. 2003, in A Massive Star Odyssey: From Main Sequence to Supernova, eds. K. van der Hucht, A. Herrero, \& C. Esteban, IAU Symp., 212, 148

Abbott, B. P., Abbott, R., Abbott, T. D., et al. 2017, ApJ, 848, L13

Adams, S. M., Kochanek, C. S., Gerke, J. R., Stanek, K. Z., \& Dai, X. 2017, MNRAS, 468, 4968

Bailer-Jones, C. A. L. 2015, PASP, 127, 994

Bailer-Jones, C. A. L., Rybizki, J., Fouesneau, M., Mantelet, G., \& Andrae, R. 2018, AJ, 156, 58

Bassino, L. P., Dessaunet, V. H., Muzzio, J. C., \& Waldhausen, S. 1982, MNRAS, 201, 885

Bersten, M. C., Benvenuto, O. G., Folatelli, G., et al. 2014, AJ, 148, 68

Bodenheimer, P., \& Woosley, S. E. 1983, ApJ, 269, 281

Boian, I., \& Groh, J. H. 2018, A\&A, 617, A115

Brott, I., de Mink, S. E., Cantiello, M., et al. 2011, A\&A, 530, A115

Brown, J. M., \& Woosley, S. E. 2013, ApJ, 769, 99

Castor, J. I., Abbott, D. C., \& Klein, R. I. 1975, ApJ, 195, 157

Chieffi, A., \& Limongi, M. 2013, ApJ, 764, 21

Conti, P. S. 1976, Proc. 20th Colloq. Int. Ap. Liège, University of Liège, 132, 193

Crowther, P. A., De Marco, O., \& Barlow, M. J. 1998, MNRAS, 296, 367

Crowther, P. A., Dessart, L., Hillier, D. J., Abbott, J. B., \& Fullerton, A. W. 2002, A\&A, 392, 653

Cui, Z., Wang, Z., Zhu, C., et al. 2018, PASP, 130, 084202

De Becker, M., \& Raucq, F. 2013, A\&A, 558, A28

Demers, H., Moffat, A. F. J., Marchenko, S. V., Gayley, K. G., \& Morel, T. 2002, ApJ, 577, 409

Desforges, S., St-Louis, N., Chené, A.-N., de la Chevrotière, A., \& HénaultBrunet, V. 2017, MNRAS, 465, 1227

Dessart, L., Hillier, D. J., Li, C., \& Woosley, S. 2012, MNRAS, 424, 2139

Dessart, L., John Hillier, D., Yoon, S.-C., Waldman, R., \& Livne, E. 2017, A\&A, 603, A51

Drissen, L., Robert, C., \& Moffat, A. F. J. 1992, ApJ, 386, 288

Ekström, S., Georgy, C., Eggenberger, P., et al. 2012, A\&A, 537, A146

Eldridge, J. J., \& Stanway, E. R. 2009, MNRAS, 400, 1019

Eldridge, J. J., Fraser, M., Maund, J. R., \& Smartt, S. J. 2015, MNRAS, 446, 2689

Eldridge, J. J., Stanway, E. R., Xiao, L., et al. 2017, PASA, 34, e058

Frey, L. H., Fryer, C. L., \& Young, P. A. 2013, ApJ, 773, L7

Gaia Collaboration (Prusti, T., et al.) 2016, A\&A, 595, A1

Gaia Collaboration (Brown, A. G. A., et al.) 2018, A\&A, 616, A1

Gal-Yam, A., Fox, D. B., Kulkarni, S. R., et al. 2005, ApJ, 630, L29

Georgy, C., Ekström, S., Meynet, G., et al. 2012, A\&A, 542, A29

Glatzel, W. 2008, in Hydrogen-Deficient Stars, eds. A. Werner, \& T. Rauch, ASP

Conf. Ser., 391, 307

Götberg, Y., de Mink, S. E., Groh, J. H., et al. 2018, A\&A, 615, A78

Gräfener, G., \& Hamann, W.-R. 2005, A\&A, 432, 633 


\section{A. A. C. Sander et al.: The Galactic WC and WO stars}

Gräfener, G., \& Hamann, W.-R. 2008, A\&A, 482, 945

Gräfener, G., \& Vink, J. S. 2013, A\&A, 560, A6

Gräfener, G., Vink, J. S., de Koter, A., \& Langer, N. 2011, A\&A, 535, A56

Gräfener, G., Owocki, S. P., \& Vink, J. S. 2012a, A\&A, 538, A40

Gräfener, G., Vink, J. S., Harries, T. J., \& Langer, N. 2012b, A\&A, 547, A83

Gräfener, G., Owocki, S. P., Grassitelli, L., \& Langer, N. 2017, A\&A, 608, A34

Grassitelli, L., Langer, N., Grin, N. J., et al. 2018, A\&A, 614, A86

Groh, J. H., Oliveira, A. S., \& Steiner, J. E. 2008, A\&A, 485, 245

Groh, J. H., Meynet, G., Georgy, C., \& Ekström, S. 2013, A\&A, 558, A131

Groh, J. H., Meynet, G., Ekström, S., \& Georgy, C. 2014, A\&A, 564, A30

Hachinger, S., Mazzali, P. A., Taubenberger, S., et al. 2012, MNRAS, 422, 70

Hainich, R., Rühling, U., Todt, H., et al. 2014, A\&A, 565, A27

Hainich, R., Pasemann, D., Todt, H., et al. 2015, A\&A, 581, A21

Hamann, W., \& Gräfener, G. 2004, A\&A, 427, 697

Hamann, W., \& Koesterke, L. 1998, A\&A, 335, 1003

Hamann, W.-R., Gräfener, G., \& Koesterke, L. 2003, in A Massive Star Odyssey: From Main Sequence to Supernova, eds. K. van der Hucht, A. Herrero, \& C. Esteban, IAU Symp., 212, 198

Hamann, W., Gräfener, G., \& Liermann, A. 2006, A\&A, 457, 1015

Heger, A., \& Langer, N. 2000, ApJ, 544, 1016

Herald, J. E., Hillier, D. J., \& Schulte-Ladbeck, R. E. 2001, ApJ, 548, 932

Hiltner, W. A., \& Schild, R. E. 1966, ApJ, 143, 770

Hjorth, J., Sollerman, J., Møller, P., et al. 2003, Nature, 423, 847

Humphreys, R. M., Weis, K., Davidson, K., \& Gordon, M. S. 2016, ApJ, 825, 64

Ishii, M., Ueno, M., \& Kato, M. 1999, PASJ, 51, 417

Johnson, S. A., Kochanek, C. S., \& Adams, S. M. 2017, MNRAS, 472, 3115

Krtička, J., \& Kubát, J. 2017, A\&A, 606, A31

Kudritzki, R. P., Pauldrach, A., Puls, J., \& Abbott, D. C. 1989, A\&A, 219, 205

Kudritzki, R. P., Springmann, U., Puls, J., Pauldrach, A. W. A., \& Lennon, M. 1998, in Properties of Hot Luminous Stars, ed. I. Howarth, ASP Conf. Ser., 131, 299

Kudritzki, R. P., Puls, J., Lennon, D. J., et al. 1999, A\&A, 350, 970

Langer, N. 1989, A\&A, 210, 93

Langer, N. 1992, A\&A, 265, L17

Langer, N., Hamann, W.-R., Lennon, M., et al. 1994, A\&A, 290, 819

Liermann, A., Hamann, W.-R., Oskinova, L. M., Todt, H., \& Butler, K. 2010, A\&A, 524, A82

Lindegren, L., Lammers, U., Bastian, U., et al. 2016, A\&A, 595, A4

Lindegren, L., Hernández, J., Bombrun, A., et al. 2018, A\&A, 616, A2

Lundstrom, I., \& Stenholm, B. 1984, A\&AS, 58, 163

Luri, X., Brown, A. G. A., Sarro, L. M., et al. 2018, A\&A, 616, A9

MacFadyen, A. I., \& Woosley, S. E. 1999, ApJ, 524, 262

Maeder, A. 1987, A\&A, 178, 159

Margutti, R., Kamble, A., Milisavljevic, D., et al. 2017, ApJ, 835, 140

McClelland, L. A. S., \& Eldridge, J. J. 2016, MNRAS, 459, 1505

Meynet, G., Chomienne, V., Ekström, S., et al. 2015, A\&A, 575, A60

Muntean, V., Moffat, A. F. J., Chené, A. N., \& de La Chevrotière, A. 2009, MNRAS, 399, 1977

Muzzio, J. C. 1979, AJ, 84, 639

Nakauchi, D., \& Saio, H. 2018, ApJ, 852, 126

Nugis, T., \& Lamers, H. J. G. L. M. 2000, A\&A, 360, 227

Oskinova, L. M., \& Hamann, W. R. 2008, MNRAS, 390, L78

Oskinova, L. M., Hamann, W.-R., Feldmeier, A., Ignace, R., \& Chu, Y.-H. 2009, ApJ, 693, L44

Paczyński, B. 1967, Acta Astron., 17, 355

Petrov, B., Vink, J. S., \& Gräfener, G. 2016, MNRAS, 458, 1999

Petrovic, J., Langer, N., Yoon, S.-C., \& Heger, A. 2005, A\&A, 435, 247
Petrovic, J., Pols, O., \& Langer, N. 2006, A\&A, 450, 219

Piro, A. L., \& Morozova, V. S. 2014, ApJ, 792, L11

Prentice, S. J., Ashall, C., Mazzali, P. A., et al. 2018, MNRAS, 478, 4162 Puls, J., Vink, J. S., \& Najarro, F. 2008, A\&ARv, 16, 209

Ramachandran, V., Hamann, W.-R., Hainich, R., et al. 2018, A\&A, 615, A40

Rauw, G., Nazé, Y., Wright, N. J., et al. 2015, ApJS, 221, 1

Rosslowe, C. K., \& Crowther, P. A. 2015, MNRAS, 447, 2322

Sander, A., Hamann, W.-R., \& Todt, H. 2012, A\&A, 540, A144

Sander, A., Todt, H., Hainich, R., \& Hamann, W.-R. 2014, A\&A, 563, A89

Sander, A., Shenar, T., Hainich, R., et al. 2015, A\&A, 577, A13

Sander, A. A. C., Hamann, W.-R., Todt, H., Hainich, R., \& Shenar, T. 2017, A\&A, 603, A86

Sander, A. A. C., Fürst, F., Kretschmar, P., et al. 2018, A\&A, 610, A60

Schmutz, W., Hamann, W., \& Wessolowski, U. 1989, A\&A, 210, 236

Shenar, T., Hamann, W.-R., \& Todt, H. 2014, A\&A, 562, A118

Shenar, T., Hainich, R., Todt, H., et al. 2016, A\&A, 591, A22

Smartt, S. J. 2009, ARA\&A, 47, 63

Smartt, S. J. 2015, PASA, 32, e016

Smith, L. F. 1968a, MNRAS, 138, 109

Smith, L. F. 1968b, MNRAS, 140, 409

Smith, N., \& Conti, P. S. 2008, ApJ, 679, 1467

Smith, N., \& Owocki, S. P. 2006, ApJ, 645, L45

Smith, N., \& Tombleson, R. 2015, MNRAS, 447, 598

Smith, N., Aghakhanloo, M., \& Murphy, J. W. 2018, ArXiv e-prints [arXiv:1805.03298]

Sokal, K. R., Skinner, S. L., Zhekov, S. A., Güdel, M., \& Schmutz, W. 2010, ApJ, 715, 1327

Sukhbold, T., Ertl, T., Woosley, S. E., Brown, J. M., \& Janka, H.-T. 2016, ApJ, 821,38

Todt, H., Sander, A., Hainich, R., et al. 2015, A\&A, 579, A75

Tramper, F., Straal, S. M., Sanyal, D., et al. 2015, A\&A, 581, A110

Tramper, F., Sana, H., \& de Koter, A. 2016, ApJ, 833, 133

Tuthill, P. G., Monnier, J. D., \& Danchi, W. C. 1999, Nature, 398, 487

Usov, V. V. 1991, MNRAS, 252, 49

Vanbeveren, D., De Donder, E., van Bever, J., van Rensbergen, W., \& De Loore, C. 1998, New Astron., 3, 443

Vanbeveren, D., Van Bever, J., \& Belkus, H. 2007, ApJ, 662, L107

Van Dyk, S. D., Zheng, W., Brink, T. G., et al. 2018, ApJ, 860, 90

Vink, J. S. 2017, A\&A, 607, L8

Vink, J. S., \& de Koter, A. 2005, A\&A, 442, 587

Vink, J. S., \& Gräfener, G. 2012, ApJ, 751, L34

Vink, J. S., de Koter, A., \& Lamers, H. J. G. L. M. 2000, A\&A, 362, 295

Vink, J. S., Gräfener, G., \& Harries, T. J. 2011, A\&A, 536, L10

Williams, P. M., van der Hucht, K. A., \& Rauw, G. 2005, in Massive Stars and High-Energy Emission in OB Associations, eds. G. Rauw, Y. Nazé, R. Blomme, \& E. Gosset, 65

Williams, P. M., Crowther, P. A., \& van der Hucht, K. A. 2015, MNRAS, 449, 1834

Woosley, S. E. 1993, ApJ, 405, 273

Woosley, S. E., \& Bloom, J. S. 2006, ARA\&A, 44, 507

Woosley, S. E., \& Heger, A. 2006, ApJ, 637, 914

Yoon, S.-C. 2017, MNRAS, 470, 3970

Yoon, S.-C., \& Langer, N. 2005, A\&A, 443, 643

Yoon, S.-C., Langer, N., \& Norman, C. 2006, A\&A, 460, 199

Yoon, S.-C., Woosley, S. E., \& Langer, N. 2010, ApJ, 725, 940

Yoon, S.-C., Gräfener, G., Vink, J. S., Kozyreva, A., \& Izzard, R. G. 2012, A\&A, 544, L11 